\title{
Closure relations during the plateau emission of Swift GRBs and the fundamental plane
}

\author{
Maria Giovanna DainottI, ${ }^{1,2, *, \dagger}$ Aleksander . LenART, $^{3, \dagger}$ Nissim FraiJa, ${ }^{4}$ \\ Shigehiro Nagataki, ${ }^{5,6}$ Donald C. WarRen, ${ }^{6}$ Biagio De Simone, $^{7}$ \\ Gokul Srinivasaragavan, ${ }^{8}$ and Aurora Mata ${ }^{4}$
}

\author{
${ }^{1}$ National Astronomical Observatory of Japan, 2-21-1 Osawa, Mitaka, Tokyo 181-8588, Japan \\ ${ }^{2}$ The Graduate University for Advanced Studies, SOKENDAI, Shonankokusaimura, Hayama, Miura District, \\ Kanagawa 240-0193, Japan \\ ${ }^{3}$ Astronomical Observatory, Jagiellonian University, ul. Orla 171, 31-501 Kraków, Poland \\ ${ }^{4}$ Space Science Institute, 4765 Walnut St STE B, Boulder, C0 80301, USA \\ ${ }^{5}$ RIKEN Cluster for Pioneering Research, Astrophysical Big Bang Laboratory (ABBL), 2-1 Hirosawa, Wakō, \\ Saitama 351-0198, Japan \\ ${ }^{6}$ RIKEN Interdisciplinary Theoretical and Mathematical Sciences (iTHEMS) Program, Wakō, Saitama \\ 351-0198, Japan \\ ${ }^{7}$ Università degli Studi di Salerno, Dipartimento di Fisica “E. R. Caianiello," Fisciano, Salerno, Via Giovanni \\ Paolo II, 132, 84084, Italy \\ ${ }^{8}$ Cahill Center for Astrophysics, California Institute of Technology, 1200 E. California Blvd., Pasadena, CA \\ 91125, USA \\ *E-mail: maria.dainotti@nao.ac.jp
}

$\dagger$ The first and second authors contributed equally to this paper.

Received 2021 March 19; Accepted 2021 May 20

\begin{abstract}
The Neil Gehrels Swift observatory observes gamma-ray burst (GRB) plateaus in X-rays. We test the reliability of the closure relations through the fireball model when dealing with GRB plateau emissions. We analyze 455 X-ray light curves collected by Swift from 2005 January until 2019 August for which the redshifts are either known or unknown using the phenomenological Willingale 2007 model. Using these fits, we analyze the emission mechanisms and astrophysical environments of these GRBs through the closure relations within the time interval of the plateau emission. Finally, we test the three-dimensional fundamental plane relation (Dainotti relation) which connects the prompt peak luminosity, the time at the end of the plateau (rest frame), and the luminosity at that time, for the GRBs with redshift, concerning groups determined by the closure relations. This allows us to check if the intrinsic scatter $\sigma_{\text {int }}$ of any of these groups is reduced compared to previous literature. The most fulfilled environments for the electron spectral distribution, $p>2$, are wind slow cooling (SC) and interstellar material (ISM) slow cooling for cases in which the parameter $q$, which indicates the flatness of the plateau emission and accounts for the energy injection, is 0 and 0.5 , respectively, in cases with both known and unknown redshifts. We also find that for short GRBs all ISM environments with $q=0$ have the smallest $\sigma_{\text {int }}=0.04 \pm 0.15$ in terms of the fundamental plane relation holding a probability of occurring by chance of $p=0.005$. We have shown that the majority of GRBs
\end{abstract}


presenting plateau emission fulfill the closure relations, including the energy injection, with a particular preference for the wind SC environment. The subsample of GRBs that fulfill the given relations can be used as possible standard candles and can suggest a way to reduce the intrinsic scatter of these studied relationships.

Key words: gamma-ray bursts: general — radiation mechanisms: non-thermal —X-rays: ISM

\section{Introduction}

Gamma-ray bursts (GRBs) are the furthest and most explosive objects witnessed by humanity so far. GRBs are observed from gamma-rays to sometimes radio wavelengths (for a review, see Kumar \& Zhang 2015). Their energy emission mechanisms have been widely studied since their discovery. One satellite that has been foremost in studying GRBs is the Neil Gehrels Swift Observatory (Gehrels et al. 2004) that has discovered many GRBs from low to high redshifts, with almost of all of them possessing an afterglow emission. The afterglow is a long-lasting (from minutes, hours, days, to even months and years) emission observed in several wavelengths from gamma-ray to radio (see, e.g., Sari \& Piran 1999b; Kumar \& Piran 2000; Panaitescu et al. 2001; Soderberg et al. 2006; Izzo et al. 2013; Stratta et al. 2013; Fraija 2015; Fraija et al. 2020b). It most probably comes from an external forward shock (ES), where the GRB ejecta that move at relativistic speeds, made up of electrons and positrons $\left(e^{-}, e^{+}\right)$and heavier nuclei, interact with the interstellar medium (Paczynski \& Rhoads 1993; Meszaros \& Rees 1994, 1997; Sari \& Piran 1995). The ES model can be verified through relationships, based on theory, called closure relations (CRs), which relate the afterglow's temporal index $(\alpha)$ considering a power law (PL) and the spectral index $(\beta)$ due to synchrotron emission (Sari et al. 1998; Panaitescu $\&$ Kumar 2002). Furthermore, each of these relations indicates different possible environments from which the GRBs stem. The Swift GRB light curves (LCs) in many cases also include a plateau emission (PE), a flat portion of the LC, which follows the prompt emission decays (Nousek et al. 2006; O’Brien et al. 2006; Zhang et al. 2006; Sakamoto et al. 2007; Zhao et al. 2019). The interpretations related to PE vary from an energy injection model due to the central engine (Dai \& Lu 1998a; Rees \& Mészáros 1998; Panaitescu et al. 1998; Sari \& Mészáros 2000; Zhang \& Mészáros 2001; Zhang et al. 2006; Liang et al. 2007), to a magnetar (e.g., Zhang \& Mészáros 2001; Toma et al. 2007; Troja et al. 2007; Dall'Osso et al. 2011; Rowlinson et al. 2013, 2014; Rea et al. 2015; Beniamini \& Mochkovitch 2017; Stratta et al. 2018; Metzger et al. 2018; Fraija et al. 2020c), or mass fall-back accretion onto a black hole (Kumar et al. 2008; Cannizzo \& Gehrels 2009; Cannizzo et al. 2011; Beniamini et al. 2017; Metzger et al.
2018). We stress here that evolving microphysical parameters (Fan \& Piran 2006; Panaitescu et al. 2006) and the off-axis jet scenario (Fraija et al. 2019, 2020a) have been employed to interpret the PE phase. Additional models regarding a structured jet have also been presented (Ito et al. 2014; Beniamini \& Mochkovitch 2017).

Within the cosmological context, GRBs can potentially be used as standardizable candles, because they are detected up to redshift $z \sim 9$. The most popular standard candles are Type Ia supernovae ( $\mathrm{SNe} \mathrm{Ia}$ ), but the problem is that they can be observed only for $z \lesssim 3$ (Rodney et al. 2015). Therefore, using GRBs for the same purpose would allow astronomers to cover orders of magnitude on the distance ladder problem. However, the prompt luminosities of GRBs range over eight orders of magnitude, which is why trying to analyze the entire set of GRBs as a whole has proven difficult, especially because many of them have fundamentally different intrinsic physics. Dainotti et al. (2010) classified GRBs that share similarities considering their phenomenological properties, and such a morphological classification can lead to more reliable correlations (Cardone et al. 2009, 2010; Dainotti et al. 2016, 2017a, 2017b; Dainotti \& Del Vecchio 2017). An attempt to check if the two-dimensional (2D) Dainotti relation is independent of the $\alpha$ parameter after the PE by using Willingale et al. (2007) is provided by Del Vecchio, Dainotti, and Ostrowski (2016). Dainotti et al. (2016) built a tight three-dimensional (3D) fundamental plane relation and pinpointed a subsample of GRBs called the "Gold" class which forms a tight fundamental plane between the luminosity at the end of the PE in the afterglow, the time at the end of the PE in the afterglow, and the peak luminosity within $1 \mathrm{~s}$ of the prompt emission: $\log \left(T_{\mathrm{a}}\right)$ $\log \left(L_{\text {peak }}\right)-\log \left(L_{\mathrm{a}}\right)$. This relationship is called the Dainotti 3D relation (where for simplicity of notation we denote $T_{a}=T_{a}^{*}$, the rest-frame time at the end of the PE), and is an extension of the Dainotti 2D relations $\log \left(T_{\mathrm{a}}\right)-\log \left(L_{\mathrm{a}}\right)$ (Dainotti et al. 2008, 2011a, 2013, 2015a, 2017b) and $\log \left(L_{\text {peak }}\right)-\log \left(L_{\mathrm{a}}\right)$ (Dainotti et al. 2011b, 2015b). There are several interpretations of the Dainotti 3D relation starting from the 2D relation. It can be explained within several scenarios: an accretion model on a black hole (Cannizzo et al. 2011), the supercritical pile model (Kazanas et al. 2015), and the magnetar model (Rowlinson et al. 2014; Rea et al. 
Table 1. CRs studied in this paper similarly to the investigations of Zhang et al. (2006).*

\begin{tabular}{lcccc}
\hline & $v$ & $\beta(p)$ & $\alpha(p)$ & $\alpha(\beta)(p>2)$ \\
\hline ISM, SC & $v_{\mathrm{m}}<v<v_{\mathrm{c}}$ & $\frac{p-1}{2}$ & $\alpha=\frac{(2 p-6)+(p+3) q}{4}$ & $\alpha=(q-1)+\frac{(2+q) \beta}{2}$ \\
& $v>v_{\mathrm{c}}$ & $\frac{p}{2}$ & $\alpha=\frac{(2 p-4)+(p+2) q}{4}$ & $\alpha=\frac{(q-2)}{2}+\frac{(2+q) \beta}{2}$ \\
ISM, FC & $v>v_{\mathrm{m}}$ & $\frac{p}{2}$ & $\alpha=\frac{(2 p-4)+(p+2) q}{4}$ & $\alpha=\frac{q-2}{2}+\frac{(2+q) \beta}{2}$ \\
Wind, SC & $v_{\mathrm{m}}<v<v_{\mathrm{c}}$ & $\alpha=\frac{(2 p-2)+(p+1) q}{4}$ & $\alpha=\frac{q}{2}+\frac{(2+q) \beta}{2}$ \\
& $v>v_{\mathrm{c}}$ & $\frac{p}{2}$ & $\alpha=\frac{(2 p-4)+(p+2) q}{2}$ & $\alpha=\frac{q-2}{2}+\frac{(2+q) \beta}{2}$ \\
Wind, FC & $v>v_{\mathrm{m}}$ & $\frac{p}{2}$ & $\alpha=\frac{(2 p-4)+(p+2) q}{4}$ & $\alpha=\frac{q-2}{2}+\frac{(2+q) \beta}{2}$ \\
\hline
\end{tabular}

*The first column contains the frequency ranges, the second the spectral index, and the third and fourth the temporal index for $p>2$.

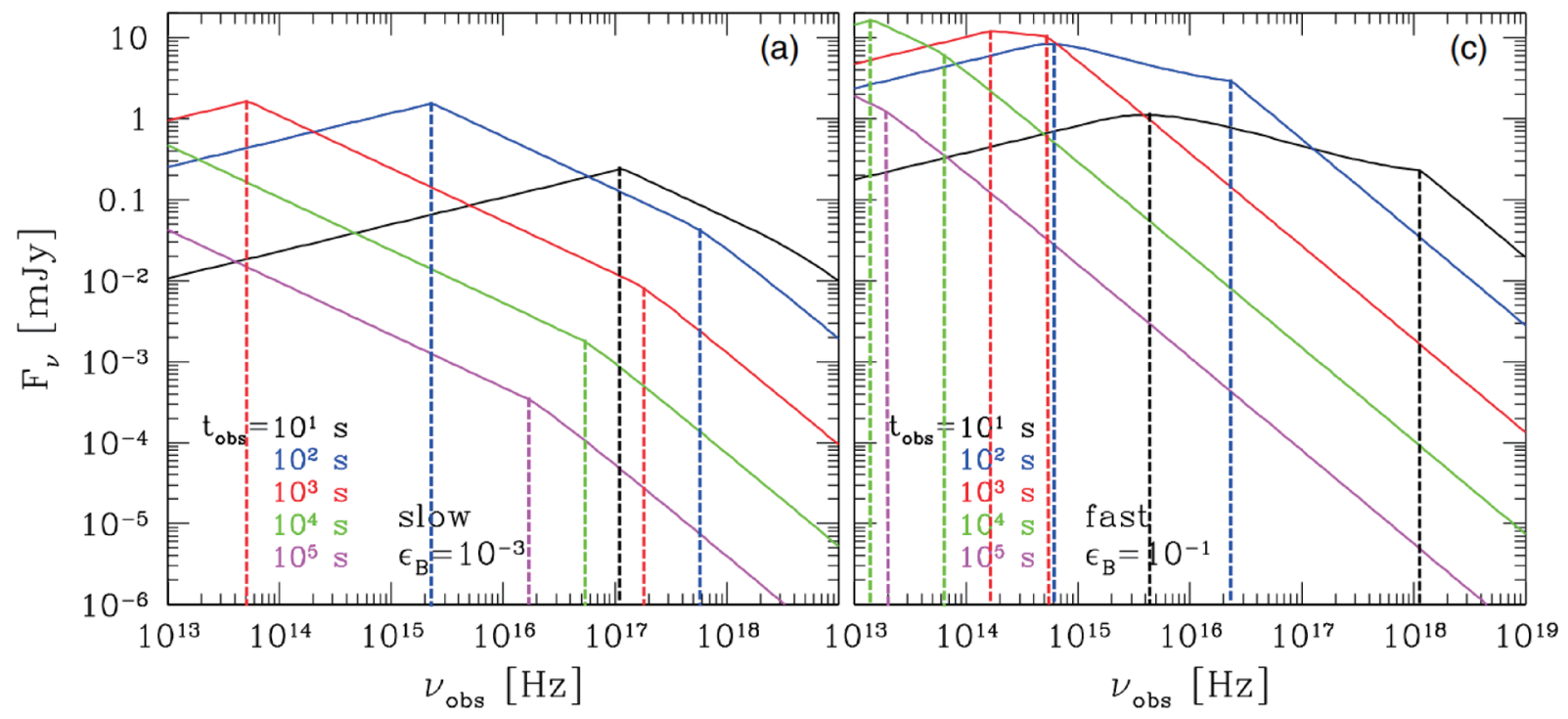

Fig. 1. Modified figure 3 from Uhm and Zhang (2014) in mJy for the flux, representing afterglow spectra in the ES corresponding to a range of times, in both the SC and FC cases, in a constant ISM medium, and showing the breaks with dashed lines. (Color online)

2015; Stratta et al. 2018), where the luminosity of an ultramagnetized millisecond pulsar is supposed to generate the plateau emission.

Our main goals are the following:

(1) to investigate the PE phase of Swift GRBs considering their emission mechanisms and progenitor environments from an astrophysical point of view for both short and long GBRs (sGRBs and lGRBs);

(2) to pinpoint, if it exists, a subclass of GRBs with peculiar features whose intrinsic scatter from the fundamental plane correlations is reduced. The aim is to help further the process of understanding GRBs to create a new type of standard candle from a cosmological point of view. In this case we also divide GRBs into sGRBs and lGRBs.

To achieve goal 1, we try out several CRs described in Racusin et al. (2009) for 455 GRB LCs observed by Swift (for which the redshift is either known or unknown) from the beginning of 2005 until the end of 2019 August. This work, thus, covers $15 \mathrm{yr}$ of detections, using the spectral indices $\beta$ corresponding to the PE duration, and brings new insight into the emission processes of GRBs.

To achieve goal 2, we divide GRBs into groups according to the astrophysical environments they belong to. These groups are built depending on whether GRBs fulfill the CRs. Additionally, we check the 3D Dainotti relation for these environments to see if the intrinsic scatter decreases in comparison to past literature. Furthermore, we make a comparison between our results and those reported in Srinivasaragavan et al. (2020), who did a similar analysis but using a different time, namely the time after the end of the PE.

Energy injection (Dai \& Lu 1998a, 1998b; Panaitescu et al. 1998; Sari \& Mészáros 2000; Zhang \& Mészáros 2001; Zhang et al. 2006; Lü \& Zhang 2014; Lü et al. 2015; Chen et al. 2017; Zhao et al. 2020; Ma et al. 2021) and emission at high latitude (Kumar \& Panaitescu 2000; O'Brien et al. 2006; Genet \& Granot 2009; Willingale et al. 2010; Zhang et al. 2011; Ascenzi et al. 2020) are the most acknowledged theoretical frameworks for PE. Here, we test the CRs within the energy injection scenario. During 


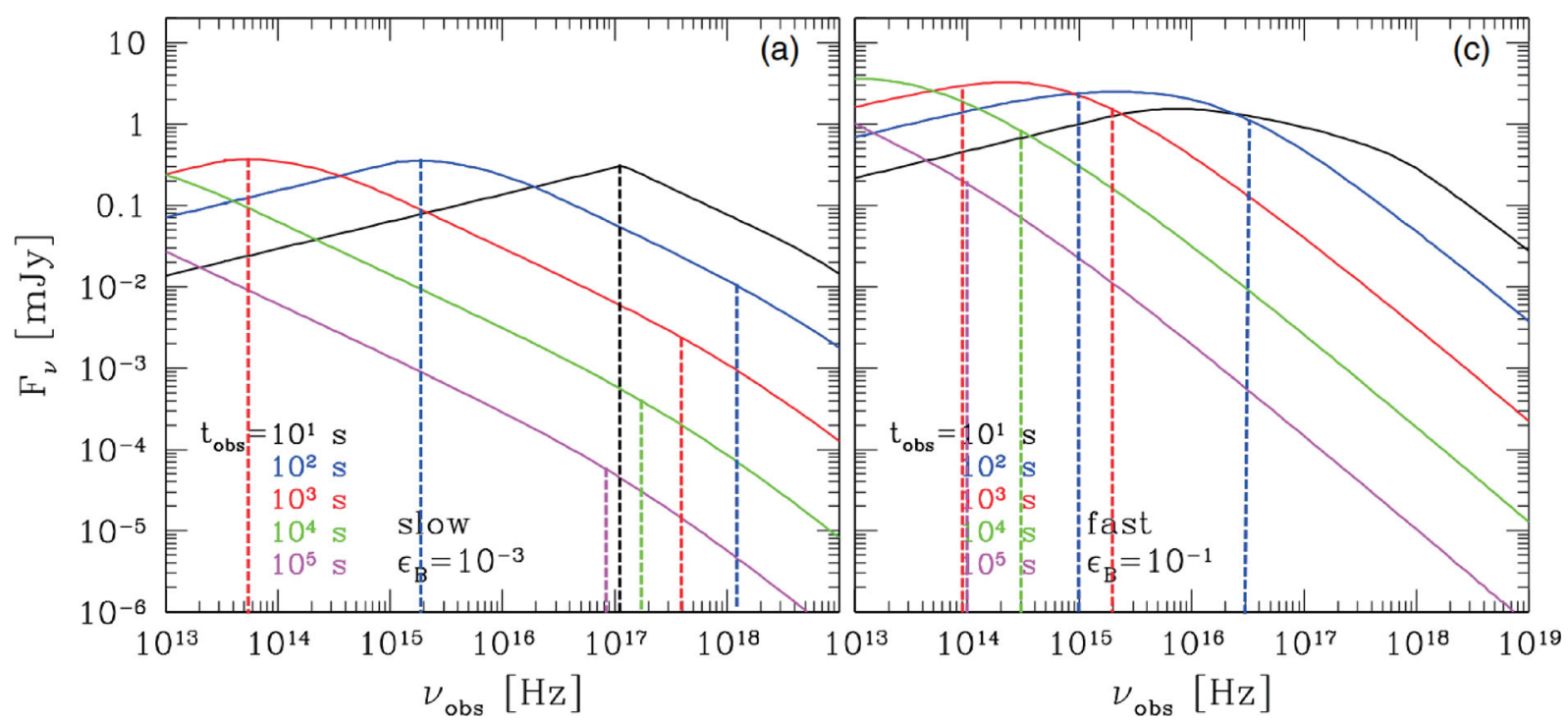

Fig. 2. Modified figure 4 from Uhm and Zhang (2014) for the flux, representing afterglow spectra in the ES corresponding to a range of times, in both the $\mathrm{SC}$ and $\mathrm{FC}$ cases for the wind medium. (Color online)

Table 2. CRs as in table 1 , but with specific values of $q=0$ and $q=0.5$

\begin{tabular}{|c|c|c|c|c|c|}
\hline & $v$ & $\beta(p)$ & $\alpha(p)$ & $\alpha(\beta)(q=0)$ & $\alpha(\beta)(q=0.5)$ \\
\hline ISM, SC & $\begin{array}{c}v_{\mathrm{m}}<v<v_{\mathrm{c}} \\
v>v_{\mathrm{c}}\end{array}$ & $\frac{\frac{p-1}{2}}{\frac{p}{2}}$ & $\begin{array}{l}\alpha=\frac{p-3}{2} \\
\alpha=\frac{p-2}{2}\end{array}$ & $\begin{array}{l}\alpha=\beta-1 \\
\alpha=\beta-1\end{array}$ & $\begin{array}{l}\alpha=\frac{5 \beta}{4}-\frac{1}{2} \\
\alpha=\frac{5 \beta}{4}-\frac{3}{4}\end{array}$ \\
\hline ISM, FC & $v>v_{\mathrm{m}}$ & $\frac{p}{2}$ & $\alpha=\frac{p-2}{2}$ & $\alpha=\beta-1$ & $\alpha=\frac{5 \beta}{4}-\frac{3}{4}$ \\
\hline Wind, SC & $\begin{array}{c}v_{\mathrm{m}}<v<v_{\mathrm{c}} \\
v>v_{\mathrm{c}}\end{array}$ & $\frac{\frac{p-1}{2}}{\frac{p}{2}}$ & $\begin{array}{l}\alpha=\frac{p-1}{2} \\
\alpha=\frac{p-2}{2}\end{array}$ & $\begin{array}{c}\alpha=\beta \\
\alpha=\beta-1\end{array}$ & $\begin{array}{l}\alpha=\frac{5 \beta}{4}+\frac{1}{4} \\
\alpha=\frac{5 \beta}{4}-\frac{3}{4}\end{array}$ \\
\hline Wind, FC & $v>v_{\mathrm{m}}$ & $\frac{p}{2}$ & $\alpha=\frac{p-2}{2}$ & $\alpha=\beta-1$ & $\alpha=\frac{5 \beta}{4}-\frac{3}{4}$ \\
\hline
\end{tabular}

the deceleration phase, a continuous energy injection may contribute to the forward shock, preventing it from decelerating as quickly as it would in the framework of impulsive energy injection. When the engine is a long-lasting one, its luminosity can be described by

$\mathcal{L}(t)=\mathcal{L}_{0}\left(\frac{t}{t_{B}}\right)^{-q}$,

whereas $\mathcal{L}_{0}$ is the luminosity at the beginning of the $\mathrm{PE}$ phase, $t_{B}$ is the transitional time between the different phases of an LC, and $q$ indicates the flatness of the PE phase. In general, for the injection mechanism to change the blast-wave dynamics the condition $q<1$ is required (Zhang et al. 2006). Thus, in this work we will use this particular assumption and we will not discuss the cases for $q \geq 1$. Here, we consider the cases of $q=0$ and $q=0.5$. We stress that for $q=1$, the standard synchrotron forward-shock model is recovered (Sari et al. 1998), but $q=1$ means that we have instantaneous energy injection.

\section{Data set and methodology}

The data set and methodology used follow closely the procedures detailed in Srinivasaragavan et al. (2020) and Dainotti et al. (2020). Regarding the spectral analysis, we computed a time-window spectrum for every particular GRB present in our sample during the PE. From the beginning until the end of the PE, denoted by $T_{\mathrm{t}}$ and $T_{\mathrm{a}}$ respectively, we use the Swift BAT+XRT online repository. ${ }^{1}$ We calculate the photon index by averaging values from the windowed and the photon-counting modes of the XRT. We discard GRBs that have $\delta_{\beta} / \beta>1 / 2$ and $\delta_{\alpha} / \alpha>1 / 2$, where $\delta_{\alpha}$ and $\delta_{\beta}$ are the error bars of $\alpha$ and $\beta$. The discarded GRBs correspond to GRBs whose error bars are larger than $50 \%$ of the measurement itself. We additionally discard GRBs which have spectral indexes with an extremely high value $(>6)$. The spectral index can be written as $\beta=\Gamma-1$, where $\Gamma$ is the photon index.

Originally, GRBs were classified solely using their duration as either short (sGRBs, $T_{90} \leq 2 \mathrm{~s}$; Mazets et al. 1981;

1 〈https://www.swift.ac.uk/xrt_spectra/〉. 

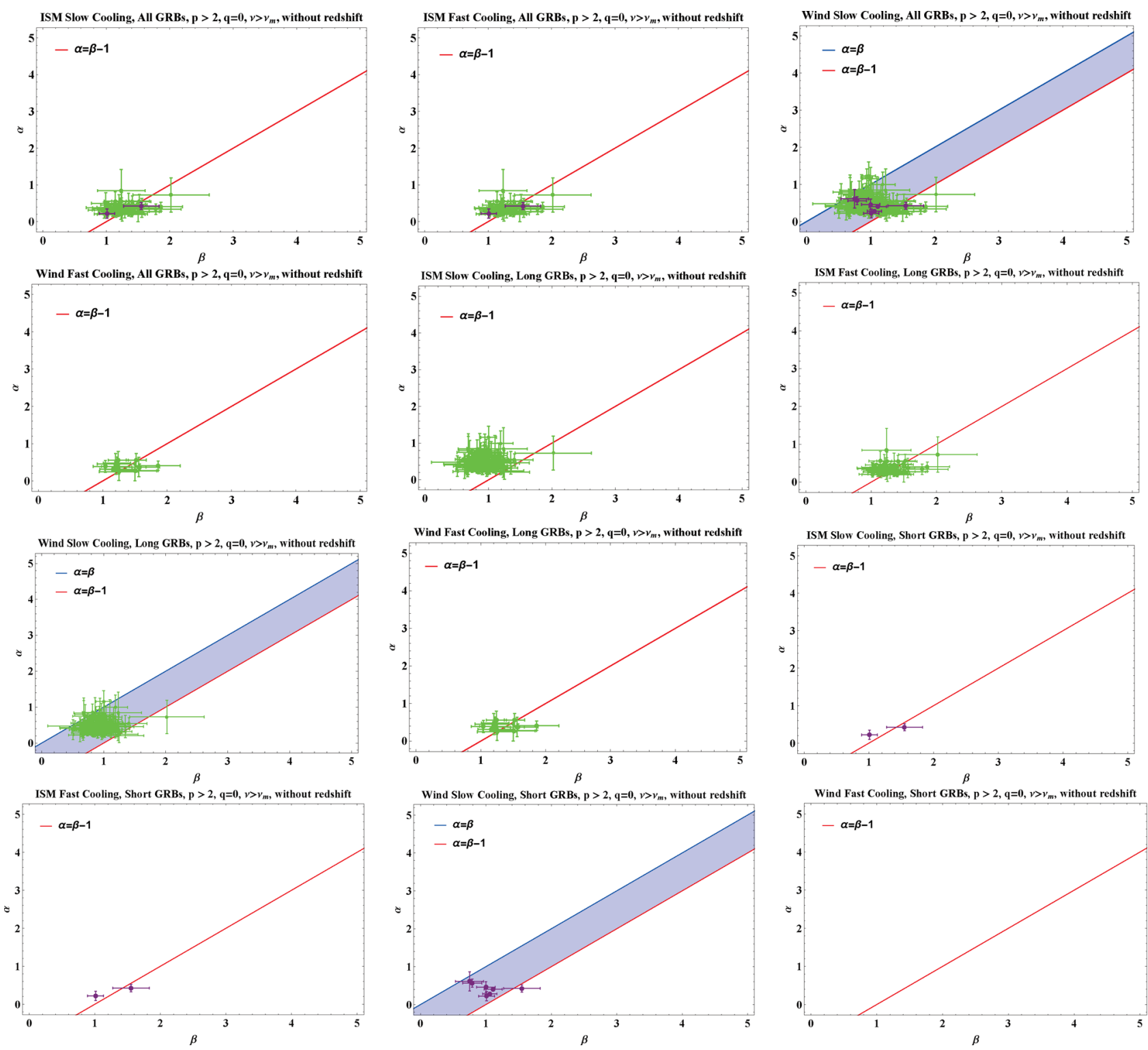

Fig. 3. Compatible GRBs for CRs in the case $q=0, v>v_{\mathrm{m}}, p>2$ without redshift. In the first four panels, the IGRB and sGRB classes are gathered together, while in the latter eight panels these classes are separated (however, the fourth panel presents no sGRBs). From this figure on, green markers will represent IGRBs while purple markers the sGRBs. We stress that the colored lines indicate the CRs and the darker sectors mark the compatibility region of GRBs with CRs. (Color online)

Kouveliotou et al. 1993) or long (lGRBs, $\left.T_{90}>2 \mathrm{~s}\right)$, a classification that is independent of energy ranges and instruments (Qin et al. 2013; Zhang et al. 2014). As more GRBs began to be discovered, the number of classification groups grew. Some sGRBs display extended emission, and are classified as "short with extended emission" (Norris \& Bonnell 2006; Levan et al. 2007; Norris et al. 2010). It is worth noting that some $1 \mathrm{GRB}$ show an X-ray fluence greater than the gamma-ray fluence, and are thus classified as Xray flashes (XRFs). Furthermore, some GRBs show a clear association with supernovae ( $\mathrm{SNe}$ ) and kilonovae $(\mathrm{KNe})$ and are classified as GRB-SNe and GRB-KNe. Studying the
GRB-KNe class, in particular, is important due to the correlation between sGRBs and gravitational waves. Finally, ultra-long (UL) GRBs display an atypically long duration of $T_{90} \geq 1000 \mathrm{~s}$ (Nakauchi et al. 2013; Stratta et al. 2013; Levan et al. 2014; Zhang et al. 2014). We subdivide the 222 GRBs with known redshifts according to these classes and indicate them with different symbols on the fundamental plane (see section 4). In addition to these classes, we also consider the cases of internal and external plateaus. While external plateaus are created by the deceleration of the external shock (Tang et al. 2019), internal plateaus are likely powered by the GRB magnetar central engine and 

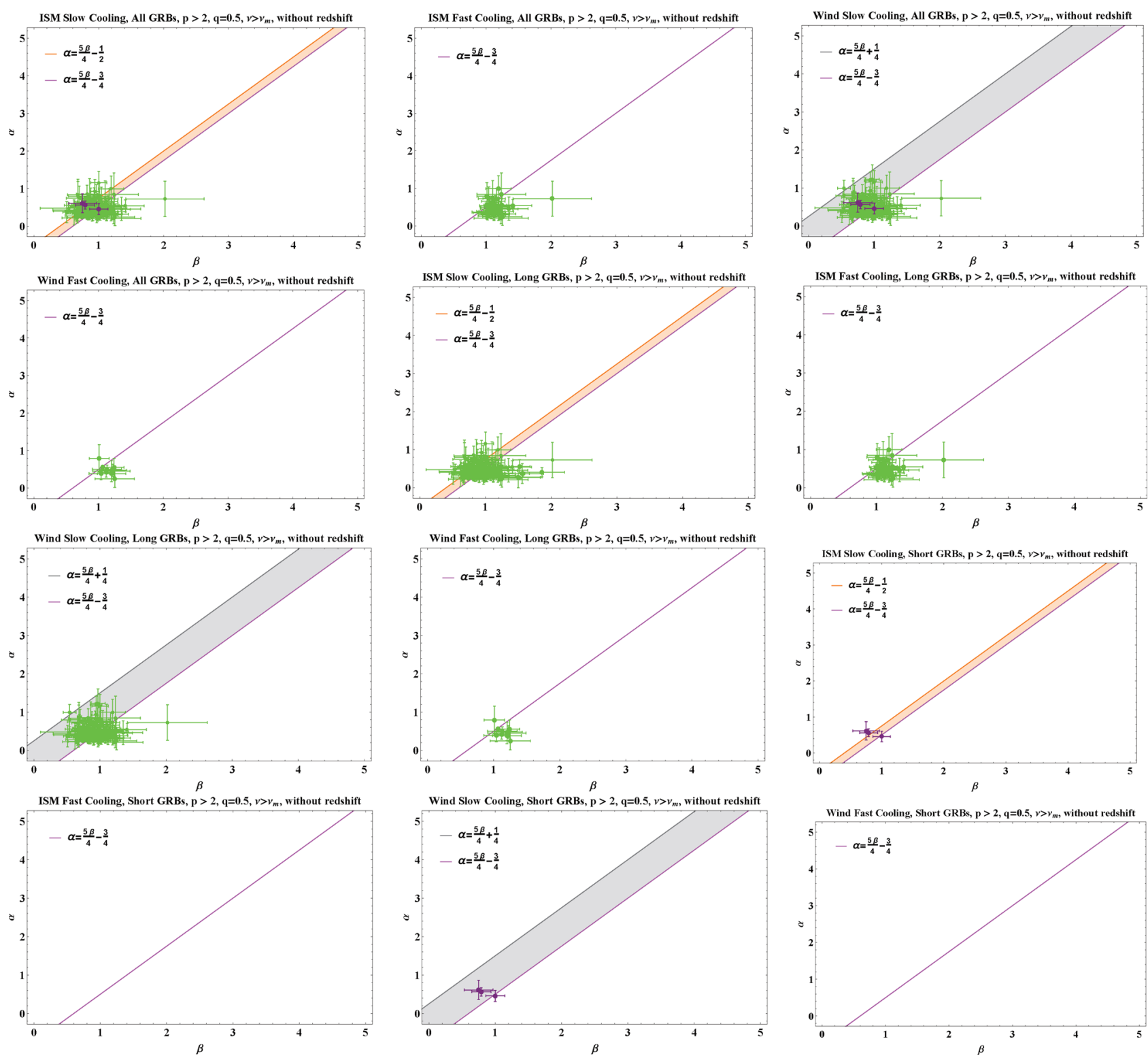

Fig. 4. Compatible GRBs for CRs in the case $q=0.5, v>v_{m}, p>2$ without redshift. In the first four panels, the IGRB and sGRB classes are gathered together, while in the latter eight panels these classes are separated. (Color online)

are characterized by values of $\alpha>(3,4)$ according to the literature (see, e.g., Li et al. 2018).

\section{Closure relations}

\subsection{Theoretical description in the context of astrophysical environments}

Following a previous approach of Lü and Zhang (2014) and Lü et al. (2015) for lGRBs and sGRBs, respectively, and of Wang et al. (2015) for the PE (phase II), we consider here the PE region instead of phase III as done in Srinivasaragavan et al. (2020), and we compare the results with phase III from our previous analysis. In this way, we have completed the analysis comprehensively during and soon after the PE region, and we can draw conclusions and comparisons about regions II and III. The aforementioned CRs consider synchrotron radiation as the main underlying mechanism of the afterglow and are computed assuming that $F_{v} \propto t^{-\alpha} v^{-\beta}$ (Sari et al. 1998). The indexes $\alpha$ and $\beta$ can be associated with the spectral index of the electron distribution, $p$, that is represented as a simple PL: $d n_{e} / d \gamma_{e} \propto \gamma_{e}^{-p}$.

We study the CRs presented in Racusin et al. (2009), where the cooling regime is the time that electrons need to cool down to the critical Lorentz factor $\gamma_{c}$, where cooling due to synchrotron radiation is significant. To test the CRs for the frequency $(v)$ and $p$ range according to table 1 , we recreate the top panels of figures 3 and 4 in the theoretical 

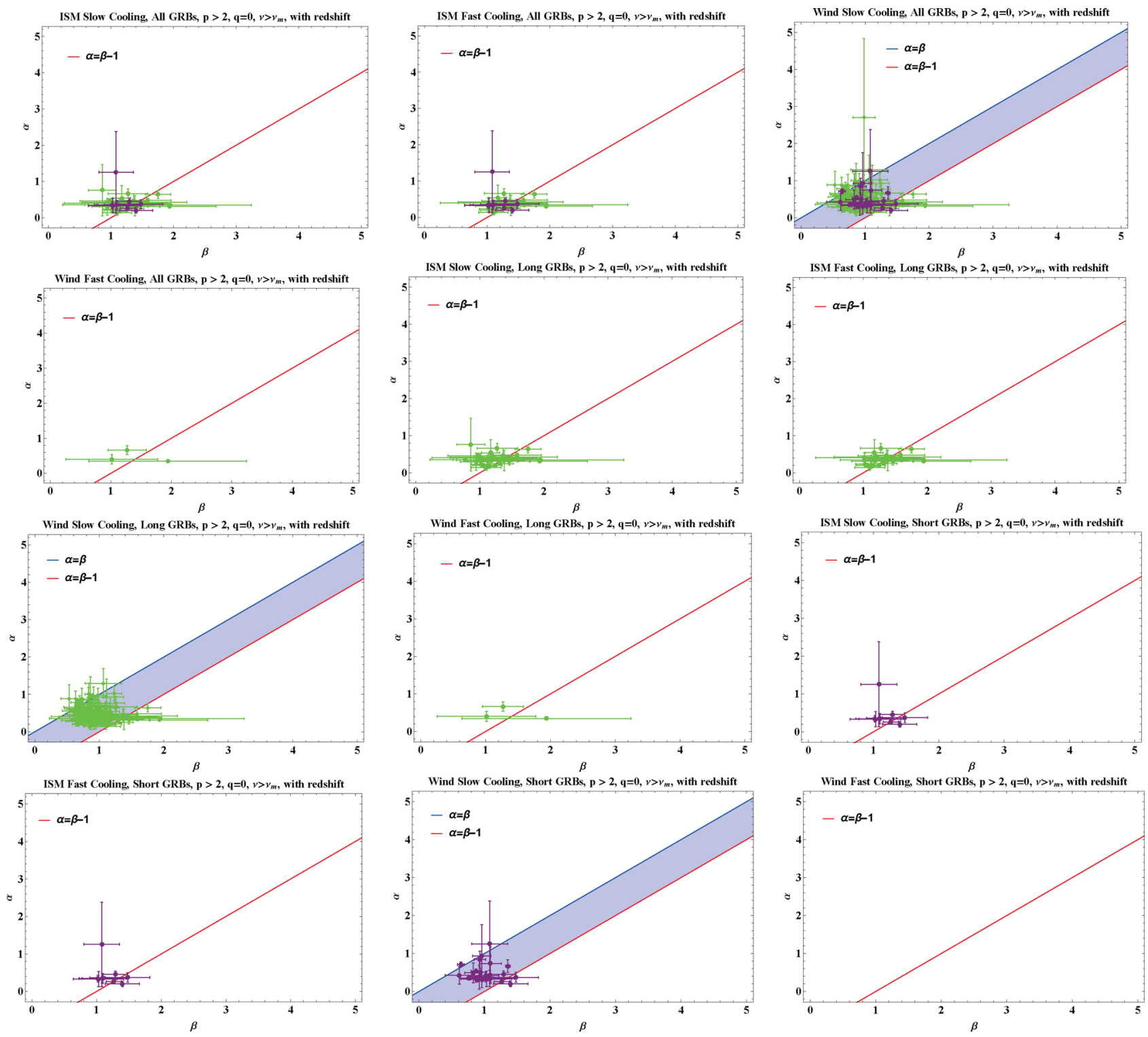

Fig. 5. Compatible GRBs for CRs in the case $q=0, v>v_{\mathrm{m}}, p>2$ where the redshift is known. In the first four panels, the IGRB and sGRB classes are gathered together (although in the fourth panel the sGRB class is empty), while in the latter eight panels these classes are separated. (Color online)

models of Uhm and Zhang (2014) and add the breaks as dashed lines corresponding to several frequencies. To match the analysis in our paper, we convert the fluxes of our fitting from erg $\mathrm{cm}^{-2} \mathrm{~s}^{-1}$ to $\mathrm{mJy}$ to check with which flux and time the spectral break corresponds (see figures 1 and 2). Then, we count how many GRBs fall within a given time and frequency range, and then we compute the $p$ values associated with these regimes and times. More specifically, we check the regime where the flux value at the end of the $\mathrm{PE}$ emission, $F_{a}$, compared to the flux values of figures 3 and 4 , can be found. Then, we subdivide every GRB into either $v_{\mathrm{m}}<v<v_{\mathrm{c}}$ and $v>v_{\mathrm{c}}$ for SC, $v>v_{\mathrm{m}}$ for fast cooling (FC). We choose $v>v_{\mathrm{m}}$ since most of the synchrotron energy is emitted in this range due to the hardness of the spectra (Nakar et al. 2009). Finally, we impose $p>$ 2 for all GRBs through the relationship between $\beta$ and $p$ given in table 1 . This choice is dictated by the fact that for $1<p<2$ the energy injection scenario is highly improbable and cumbersome in its treatment (Racusin et al. 2009).

We summarize here for clarity all the steps of our analysis:

(1) Obtain $\alpha$ and $T_{\mathrm{a}}$ by fitting the PE with the function from Willingale et al. (2007).

(2) Calculate the temporal interval region (time slice) for the PE phase by a fitting which determines $T_{\mathrm{t}}$ and $T_{\mathrm{a}}$, and calculate $\beta$ in this time slice directly from the observations. 

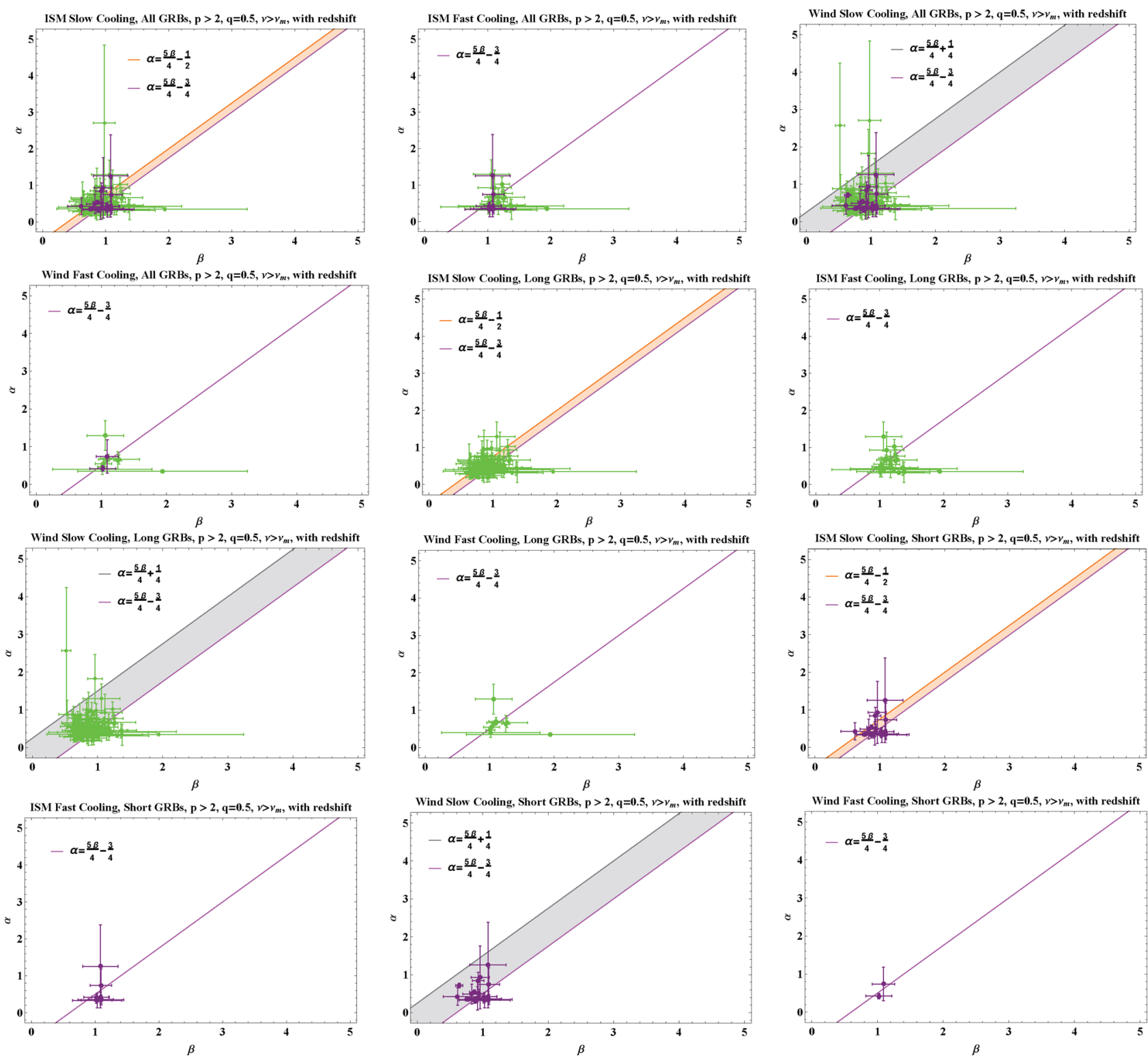

Fig. 6. Compatible GRBs for CRs in the case $q=0.5, v>v_{\mathrm{m}}, p>2$ where the redshift is known. In the first four panels, the IGRB and sGRB classes are gathered together, while in the latter eight panels these classes are separated. (Color online)

(3) Calculate the CRs.

(4) For all four combinations of FC vs. SC and ISM vs. a wind-like circumburst medium we take the following steps:

a Determine which curve in figures 1 and 2 is the closest to $T_{\mathrm{a}}$ as found in step 1 .

b For the particular curve identified in figures 1 and 2 , determine whether $v_{\mathrm{m}}\left(v_{\mathrm{c}}\right)$ is above or below the Swift window of $0.3-10 \mathrm{keV}$ in the FC or SC scenario, thus corresponding to X-ray.

c Once we know whether we are above or below the relevant spectral breaks, we can identify from tables 1 and 2 which set of CRs we will test. Then, we compute the electron spectral index $p$ using the second column of tables 1 and 2 together with $\beta$ as computed in step 2 .

d Given the value of $p>2$, once the CRs have been identified we can compare their values with $\alpha$ and $\beta$.

e Alternately, if the X-ray band falls between $v_{c}$ and $v_{\mathrm{m}}$ (regardless of $\mathrm{FC}$ vs. $\mathrm{SC}$ ) we then use the GRBs for which $\beta=1 / 2$.

After all these steps, we verify all of the CRs which correspond to the appropriate $p$ and $v$ range for all GRBs investigated here. All these steps are performed by dividing the sample into sGRBs and lGRBs, and this division is kept throughout the analysis regarding the 
Table 3. CR characteristics for the sample without redshift (in the case $v>v_{\mathrm{m}}$ and $p>2$ ).*

\begin{tabular}{|c|c|c|c|c|c|c|}
\hline & $v\left(v>v_{\mathrm{m}}\right)$ & $q(p>2)$ & CR & \# GRBs & GRBs fulfilling CRs & $\%$ fulfilling CRs \\
\hline \multicolumn{7}{|c|}{ All GRB Environments without $z$} \\
\hline \multirow[t]{2}{*}{ ISM, SC } & $v_{\mathrm{m}}<v<v_{\mathrm{c}}$ & 0 & $\alpha=\beta-1$ & 229 & 65 & $28.4 \%$ \\
\hline & $v>v_{\mathrm{c}}$ & 0 & $\alpha=\beta-1$ & & & \\
\hline ISM, FC & $v>v_{\mathrm{m}}$ & 0 & $\alpha=\beta-1$ & 226 & 60 & $26.6 \%$ \\
\hline \multirow[t]{2}{*}{ Wind, SC } & $v_{\mathrm{m}}<v<v_{\mathrm{c}}$ & 0 & $\alpha=\beta$ & 229 & 204 & $89.1 \%$ \\
\hline & $v>v_{\mathrm{c}}$ & 0 & $\alpha=\beta-1$ & & & \\
\hline Wind, FC & $v>v_{\mathrm{m}}$ & 0 & $\alpha=\beta-1$ & 76 & 14 & $18.4 \%$ \\
\hline \multirow[t]{2}{*}{ ISM, SC } & $v_{\mathrm{m}}<v<v_{\mathrm{c}}$ & 0.5 & $\alpha=\frac{5 \beta}{4}-\frac{1}{2}$ & 229 & 143 & $62.4 \%$ \\
\hline & $v>v_{\mathrm{c}}$ & 0.5 & $\alpha=\frac{5 \beta}{4}-\frac{3}{4}$ & & & \\
\hline ISM, FC & $v>v_{\mathrm{m}}$ & 0.5 & $\alpha=\frac{5 \beta}{4}-\frac{3}{4}$ & 226 & 42 & $18.6 \%$ \\
\hline \multirow[t]{2}{*}{ Wind, SC } & $v_{\mathrm{m}}<v<v_{\mathrm{c}}$ & 0.5 & $\alpha=\frac{5 \beta}{4}+\frac{1}{4}$ & 229 & 154 & $67.2 \%$ \\
\hline & $v>v_{\mathrm{c}}$ & 0.5 & $\alpha=\frac{5 \beta}{4}-\frac{3}{4}$ & & & \\
\hline Wind, FC & $v>v_{\mathrm{m}}$ & 0.5 & $\alpha=\frac{5 \beta}{4}-\frac{3}{4}$ & 76 & 10 & $13.2 \%$ \\
\hline \multicolumn{7}{|c|}{ lGRB environments without $z$} \\
\hline \multirow[t]{2}{*}{ ISM, SC } & $v_{\mathrm{m}}<v<v_{\mathrm{c}}$ & 0 & $\alpha=\beta-1$ & 220 & 63 & $28.6 \%$ \\
\hline & $v>v_{\mathrm{c}}$ & 0 & $\alpha=\beta-1$ & & & \\
\hline ISM, FC & $v>v_{\mathrm{m}}$ & 0 & $\alpha=\beta-1$ & 218 & 58 & $26.6 \%$ \\
\hline \multirow[t]{2}{*}{ Wind, SC } & $v_{\mathrm{m}}<v<v_{\mathrm{c}}$ & 0 & $\alpha=\beta$ & 22 & 196 & $89.1 \%$ \\
\hline & $v>v_{\mathrm{c}}$ & 0 & $\alpha=\beta-1$ & & & \\
\hline Wind, FC & $v>v_{\mathrm{m}}$ & 0 & $\alpha=\beta-1$ & 74 & 14 & $18.9 \%$ \\
\hline \multirow[t]{2}{*}{ ISM, SC } & $v_{\mathrm{m}}<v<v_{\mathrm{c}}$ & 0.5 & $\alpha=\frac{5 \beta}{4}-\frac{1}{2}$ & 220 & 139 & $63.2 \%$ \\
\hline & $v>v_{\mathrm{c}}$ & 0.5 & $\alpha=\frac{5 \beta}{4}-\frac{3}{4}$ & & & \\
\hline ISM, FC & $v>v_{\mathrm{m}}$ & 0.5 & $\alpha=\frac{5 \beta}{4}-\frac{3}{4}$ & 218 & 42 & $19.3 \%$ \\
\hline \multirow[t]{2}{*}{ Wind, SC } & $v_{\mathrm{m}}<v<v_{\mathrm{c}}$ & 0.5 & $\alpha=\frac{5 \beta}{4}+\frac{1}{4}$ & 220 & 150 & $68.2 \%$ \\
\hline & $v>v_{\mathrm{c}}$ & 0.5 & $\alpha=\frac{5 \beta}{4}-\frac{3}{4}$ & & & \\
\hline Wind, FC & $v>v_{\mathrm{m}}$ & 0.5 & $\alpha=\frac{5 \beta}{4}-\frac{3}{4}$ & 74 & 10 & $13.5 \%$ \\
\hline \multicolumn{7}{|c|}{ sGRB environments without $z$} \\
\hline \multirow[t]{2}{*}{ ISM, SC } & $v_{\mathrm{m}}<v<v_{\mathrm{c}}$ & 0 & $\alpha=\beta-1$ & 8 & 2 & $25 \%$ \\
\hline & $v>v_{\mathrm{c}}$ & 0 & $\alpha=\beta-1$ & & & \\
\hline ISM, FC & $v>v_{\mathrm{m}}$ & 0 & $\alpha=\beta-1$ & 7 & 2 & $28.6 \%$ \\
\hline \multirow[t]{2}{*}{ Wind, SC } & $v_{\mathrm{m}}<v<v_{\mathrm{c}}$ & 0 & $\alpha=\beta$ & 8 & 7 & $87.5 \%$ \\
\hline & $v>v_{\mathrm{c}}$ & 0 & $\alpha=\beta-1$ & & & \\
\hline Wind, FC & $v>v_{\mathrm{m}}$ & 0 & $\alpha=\beta-1$ & 1 & 0 & - \\
\hline \multirow[t]{2}{*}{ ISM, SC } & $v_{\mathrm{m}}<v<v_{\mathrm{c}}$ & 0.5 & $\alpha=\frac{5 \beta}{4}-\frac{1}{2}$ & 8 & 3 & $37.5 \%$ \\
\hline & $v>v_{\mathrm{c}}$ & 0.5 & $\alpha=\frac{5 \beta}{4}-\frac{3}{4}$ & & & \\
\hline ISM, FC & $v>v_{\mathrm{m}}$ & 0.5 & $\alpha=\frac{5 \beta}{4}-\frac{3}{4}$ & 7 & 0 & - \\
\hline \multirow[t]{2}{*}{ Wind, SC } & $v_{\mathrm{m}}<v<v_{\mathrm{c}}$ & 0.5 & $\alpha=\frac{5 \beta}{4}+\frac{1}{4}$ & 8 & 3 & $37.5 \%$ \\
\hline & $v>v_{\mathrm{c}}$ & 0.5 & $\alpha=\frac{5 \beta}{4}-\frac{3}{4}$ & & & \\
\hline Wind, FC & $v>v_{\mathrm{m}}$ & 0.5 & $\alpha=\frac{5 \beta}{4}-\frac{3}{4}$ & 1 & 0 & - \\
\hline
\end{tabular}

*The upper part of the table refers to all GRBs with $q=0$ and $q=0.5$, the middle part refers to lGRBs for $q=0$ and $q=0.5$, and the lower part considers the sGRBs, again for $q=0$ and $q=0.5$.

CRs, the values of the distributions of the $q$ parameters, and the fundamental planes corresponding to particular environments.

We then plot the CRs by showing the $\alpha$ and $\beta$ parameters with $1 \sigma_{\text {int }}$ error bars, as well as solid lines that represent the equations of the CRs (see figures 3-6), along with tables 3 and 4.

The GRB afterglow LCs are usually segregated into four segments: I, the initial steep decay; II, the PE phase; III, the adiabatic fireball deceleration with the observed decay 
Table 4. CR characteristics for the sample with known redshift (in the case $v>v_{\mathrm{m}}$ and $p>2$ ).*

\begin{tabular}{|c|c|c|c|c|c|c|}
\hline & $v\left(v>v_{\mathrm{m}}\right)$ & $q(p>2)$ & CR & \# GRBs & GRBs fulfilling CR & $\%$ fulfilling CRs \\
\hline \multicolumn{7}{|c|}{ All GRB environments without $z$} \\
\hline \multirow[t]{2}{*}{ ISM, SC } & $v_{\mathrm{m}}<v<v_{\mathrm{c}}$ & 0 & $\alpha=\beta-1$ & 209 & 39 & $18.7 \%$ \\
\hline & $v>v_{\mathrm{c}}$ & 0 & $\alpha=\beta-1$ & & & \\
\hline ISM, FC & $v>v_{\mathrm{m}}$ & 0 & $\alpha=\beta-1$ & 204 & 33 & $16.2 \%$ \\
\hline \multirow[t]{2}{*}{ Wind, SC } & $v_{\mathrm{m}}<v<v_{\mathrm{c}}$ & 0 & $\alpha=\beta$ & 209 & 135 & $64.6 \%$ \\
\hline & $v>v_{\mathrm{c}}$ & 0 & $\alpha=\beta-1$ & & & \\
\hline Wind, FC & $v>v_{\mathrm{m}}$ & 0 & $\alpha=\beta-1$ & 53 & 3 & $5.7 \%$ \\
\hline \multirow[t]{2}{*}{ ISM, SC } & $v_{\mathrm{m}}<v<v_{\mathrm{c}}$ & 0.5 & $\alpha=\frac{5 \beta}{4}-\frac{1}{2}$ & 209 & 135 & $64.6 \%$ \\
\hline & $v>v_{\mathrm{c}}$ & 0.5 & $\alpha=\frac{5 \beta}{4}-\frac{3}{4}$ & & & \\
\hline ISM, FC & $v>v_{\mathrm{m}}$ & 0.5 & $\alpha=\frac{5 \beta}{4}-\frac{3}{4}$ & 204 & 32 & $15.7 \%$ \\
\hline \multirow[t]{2}{*}{ Wind, SC } & $v_{\mathrm{m}}<v<v_{\mathrm{c}}$ & 0.5 & $\alpha=\frac{5 \beta}{4}+\frac{1}{4}$ & 209 & 159 & $76.1 \%$ \\
\hline & $v>v_{\mathrm{c}}$ & 0.5 & $\alpha=\frac{5 \beta}{4}-\frac{3}{4}$ & & & \\
\hline \multirow[t]{2}{*}{ Wind, FC } & $v>v_{\mathrm{m}}$ & 0.5 & $\alpha=\frac{5 \beta}{4}-\frac{3}{4}$ & 53 & 11 & $20.8 \%$ \\
\hline & \multicolumn{6}{|c|}{ lGRB environments with $z$} \\
\hline \multirow[t]{2}{*}{ ISM, SC } & $v_{\mathrm{m}}<v<v_{\mathrm{c}}$ & 0 & $\alpha=\beta-1$ & 177 & 30 & $16.9 \%$ \\
\hline & $v>v_{\mathrm{c}}$ & 0 & $\alpha=\beta-1$ & & & \\
\hline ISM, FC & $v>v_{\mathrm{m}}$ & 0 & $\alpha=\beta-1$ & 173 & 24 & $13.9 \%$ \\
\hline \multirow[t]{2}{*}{ Wind, SC } & $v_{\mathrm{m}}<v<v_{\mathrm{c}}$ & 0 & $\alpha=\beta$ & 177 & 159 & $89.8 \%$ \\
\hline & $v>v_{\mathrm{c}}$ & 0 & $\alpha=\beta-1$ & & & \\
\hline Wind, FC & $v>v_{\mathrm{m}}$ & 0 & $\alpha=\beta-1$ & 44 & 3 & $6.8 \%$ \\
\hline \multirow[t]{2}{*}{ ISM, SC } & $v_{\mathrm{m}}<v<v_{\mathrm{c}}$ & 0.5 & $\alpha=\frac{5 \beta}{4}-\frac{1}{2}$ & 177 & 114 & $64.4 \%$ \\
\hline & $v>v_{\mathrm{c}}$ & 0.5 & $\alpha=\frac{5 \beta}{4}-\frac{3}{4}$ & & & \\
\hline ISM, FC & $v>v_{\mathrm{m}}$ & 0.5 & $\alpha=\frac{5 \beta}{4}-\frac{3}{4}$ & 173 & 24 & $13.9 \%$ \\
\hline \multirow[t]{2}{*}{ Wind, SC } & $v_{\mathrm{m}}<v<v_{\mathrm{c}}$ & 0.5 & $\alpha=\frac{5 \beta}{4}+\frac{1}{4}$ & 177 & 137 & $77.4 \%$ \\
\hline & $v>v_{\mathrm{c}}$ & 0.5 & $\alpha=\frac{5 \beta}{4}-\frac{3}{4}$ & & & \\
\hline \multirow[t]{2}{*}{ Wind, FC } & $v>v_{\mathrm{m}}$ & 0.5 & $\alpha=\frac{5 \beta}{4}-\frac{3}{4}$ & 44 & 9 & $20.4 \%$ \\
\hline & \multicolumn{6}{|c|}{ sGRB environments with $z$} \\
\hline \multirow[t]{2}{*}{ ISM, SC } & $v_{\mathrm{m}}<v<v_{\mathrm{c}}$ & 0 & $\alpha=\beta-1$ & 31 & 9 & $29 \%$ \\
\hline & $v>v_{\mathrm{c}}$ & 0 & $\alpha=\beta-1$ & & & \\
\hline ISM, FC & $v>v_{\mathrm{m}}$ & 0 & $\alpha=\beta-1$ & 30 & 9 & $30 \%$ \\
\hline \multirow[t]{2}{*}{ Wind, SC } & $v_{\mathrm{m}}<v<v_{\mathrm{c}}$ & 0 & $\alpha=\beta$ & 31 & 29 & $93.5 \%$ \\
\hline & $v>v_{\mathrm{c}}$ & 0 & $\alpha=\beta-1$ & & & \\
\hline Wind, FC & $v>v_{\mathrm{m}}$ & 0 & $\alpha=\beta-1$ & 8 & 0 & - \\
\hline \multirow[t]{2}{*}{ ISM, SC } & $v_{\mathrm{m}}<v<v_{\mathrm{c}}$ & 0.5 & $\alpha=\frac{5 \beta}{4}-\frac{1}{2}$ & 31 & 20 & $64.5 \%$ \\
\hline & $v>v_{\mathrm{c}}$ & 0.5 & $\alpha=\frac{5 \beta}{4}-\frac{3}{4}$ & & & \\
\hline ISM, FC & $v>v_{\mathrm{m}}$ & 0.5 & $\alpha=\frac{5 \beta}{4}-\frac{3}{4}$ & 30 & 8 & $26.7 \%$ \\
\hline \multirow[t]{2}{*}{ Wind, SC } & $v_{\mathrm{m}}<v<v_{\mathrm{c}}$ & 0.5 & $\alpha=\frac{5 \beta}{4}+\frac{1}{4}$ & 31 & 21 & $67.7 \%$ \\
\hline & $v>v_{\mathrm{c}}$ & 0.5 & $\alpha=\frac{5 \beta}{4}-\frac{3}{4}$ & & & \\
\hline Wind, FC & $v>v_{\mathrm{m}}$ & 0.5 & $\alpha=\frac{5 \beta}{4}-\frac{3}{4}$ & 8 & 2 & $25 \%$ \\
\hline
\end{tabular}

*The upper part of the table refers to all GRBs with $q=0$ and $q=0.5$, the middle part refers to lGRBs with $q=0$ and $q=0.5$, and the lower part considers sGRBs, again with $q=0$ and $q=0.5$.

phase; IV, the post-jet break phase (Rhoads 1999; Sari \& Piran 1999a; Kumar \& Panaitescu 2000; Mészáros 2002; Piran 2004; Liang \& Zhang 2006; Zhang et al. 2006, 2007; Racusin et al. 2009). We consider phase II of the LCs in our analysis.
Figures 3-6 show the CRs computed within the time range $T_{\mathrm{t}}-T_{\mathrm{a}}$ in the afterglow, together with the lines representing the $\mathrm{CR}$ equations and their error bars. Relations that are within the same $p$ range, cooling regime, and astrophysical environments are grouped on the same plot, drawing a 
Table 5. Energy injection parameter $q$ computed for particular $v$ ranges with equations taken from table 1.

\begin{tabular}{|c|c|c|c|c|c|c|c|c|c|c|c|c|}
\hline $\begin{array}{l}v \\
(1)\end{array}$ & $\begin{array}{l}\text { All } \\
(2)\end{array}$ & $\begin{array}{l}q=0 \\
(3)\end{array}$ & $\begin{array}{c}q=0.5 \\
(4)\end{array}$ & $\begin{array}{c}\bar{q} \\
(5)\end{array}$ & $\begin{array}{l}\text { lGRBs } \\
(6)\end{array}$ & $\begin{array}{c}q_{\mathrm{L}}=0 \\
(7)\end{array}$ & $\begin{array}{c}q_{\mathrm{L}}=0.5 \\
(8)\end{array}$ & $\begin{array}{l}\bar{q}_{\mathrm{L}} \\
(9)\end{array}$ & $\begin{array}{l}\text { sGRBs } \\
(10)\end{array}$ & $\begin{array}{c}q_{\mathrm{S}}=0 \\
(11)\end{array}$ & $\begin{array}{c}q_{\mathrm{s}}=0.5 \\
(12)\end{array}$ & $\begin{array}{c}\bar{q}_{\mathrm{S}} \\
(13)\end{array}$ \\
\hline \multicolumn{13}{|l|}{ ISM, SC } \\
\hline $\begin{array}{l}v_{\mathrm{m}}<v<v_{\mathrm{c}} \\
\text { ISM, FC }\end{array}$ & 167 & 22 & 45 & 0.37 & 145 & 17 & 42 & 0.39 & 22 & 5 & 3 & 0.39 \\
\hline $\begin{array}{l}v>v_{\mathrm{m}} \\
\text { Wind, SC }\end{array}$ & 165 & 20 & 61 & 0.58 & 142 & 15 & 52 & 0.60 & 22 & 5 & 9 & 0.41 \\
\hline $\begin{array}{l}v_{\mathrm{m}}<v<v_{\mathrm{c}} \\
\text { Wind, FC }\end{array}$ & 167 & 26 & 5 & -0.45 & 145 & 24 & 5 & -0.43 & 22 & 2 & 0 & -0.59 \\
\hline$v>v_{\mathrm{m}}$ & 44 & 0 & 12 & 0.73 & 145 & 24 & 5 & -0.43 & 6 & 0 & 2 & 0.64 \\
\hline
\end{tabular}

Table 6. Gaussian probability density function parameter values referred to the fittings in figure 7 .* $^{*}$

\begin{tabular}{lccc}
\hline ISM SC (All) & ISM FC (All) & Wind SC (All) & Wind FC (All) \\
$q=0.37 \pm 0.27$ & $q=0.58 \pm 0.42$ & $q=-0.45 \pm 0.32$ & $q=0.73 \pm 0.27$ \\
\hline ISM SC (lGRBs) & ISM FC (lGRBs) & Wind SC (lGRBs) & Wind FC (1GRBs) \\
$q=0.39 \pm 0.28$ & $q=0.60 \pm 0.42$ & $q=-0.43 \pm 0.33$ & $q=0.76 \pm 0.28$ \\
\hline ISM SC (sGRBs) & ISM FC (sGRBs) & Wind SC (sGRBs) & Wind FC (sGRBs) \\
$q=0.26 \pm 0.20$ & $q=0.41 \pm 0.32$ & $q=-0.59 \pm 0.24$ & $q=0.41 \pm 0.43$ \\
\hline
\end{tabular}

*The results are here reported in the form $q=\mu \pm \sigma$.

"gray region" where the GRBs are regarded as consistent cases. We stress that the lines representing the closure relationships are not the best-fit lines, but are lines representing the relations themselves. We color the CRs according to the following coding: red, $\alpha=\beta-1$; blue, $\alpha=\beta$; magenta, $\alpha=(5 / 4) \beta-(3 / 4)$; gray, $(5 / 4) \beta+(1 / 4)$; and orange, $(5 / 4) \beta-(1 / 2)$.

Let's take, as an example, the case of ISM SC with $p>2$ : not only should one consider $\alpha=(q-1)+[(2+q) \beta] / 2\left(v_{\mathrm{m}}\right.$ $\left.<v<v_{\mathrm{c}}\right)$ and $\alpha=(q-2) / 2+[(2+q) \beta] / 2\left(v>v_{\mathrm{c}}\right)$, but the region between these two lines, in the $\alpha-\beta$ plane, should be considered as a "consistent" region since these in-between regimes are allowed by the model; they are shown in gray in our plots. It may happen that a given couple of CRs for the considered environment degenerate into only one line. Nevertheless, this condition does not change the compatibility.

In table 5 we present a classification that allows us to categorize GRBs in terms of the $q$ value, which can be determined naturally from the relationships among $\alpha$ and $\beta$ from table 1. Here, we derive for each GRB the value of $q$ and its error $\delta q$; according to those values, we check the compatibility of each GRB's $q$ value with the reference values $q=0$ and $q=0.5$ (see table 2). Interestingly, we note that the cases for $q \geq 1$ are few, and thus are not here considered. We also present the distribution of the $q$ parameters showing the applicability of the method (see figure 7). In this figure, we highlight the boundary condition $q=1$ through a red vertical line, thus showing all the $q$ values of our categories despite considering only the ones with $q<1$ for our purposes. The results of the histograms in figure 7 are shown in table 6. From this table, it is clear that the subdivision of all GRBs into lGRBs and sGRBs for each environment generates values of $q$ compatible within $1 \sigma$ with those corresponding to sGRBs and lGRBs gathered together. The cases with $q \geq 1$ are roughly equivalent to the impulsive case as far as the energy injection is concerned. They add all their energy very quickly, and after that may be treated as an impulsive case with a different $E_{\text {iso }}$ (for reviews, see Barkov \& Pozanenko 2011; Warren et al. 2021).

\subsection{Results and interpretations}

We test the CRs given in table 1 for the 455 GRB LCs in our sample from 2005 January until 2019 August, detailing the relations calculated for the time range of the PE from $T_{\mathrm{t}}$ to $T_{\mathrm{a}}$. Figures 3-6 detail the specific CRs, including the error bars of $\alpha$ and $\beta$ in $1 \sigma_{\text {int }}$, along with the equality lines corresponding to each CR, and tables 4 and 3 show the numbers of GRBs satisfying such relations. We group in 

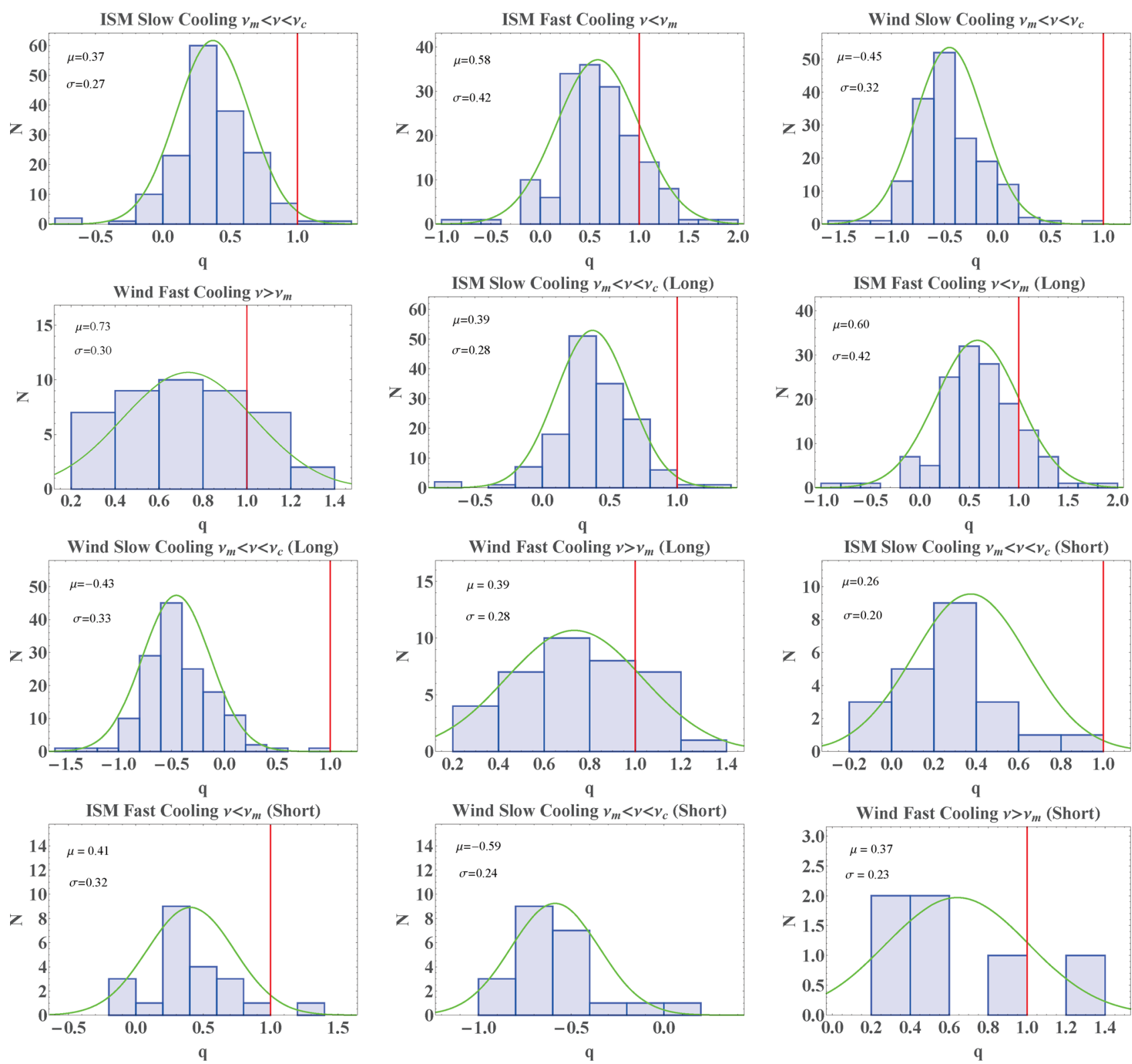

Fig. 7. According to table 5, the population of GRBs q values in each environment and category (all, IGRB, sGRB). The green curves show the Gaussian probability density function for data with mean $\mu$ and standard deviation $\sigma$. The vertical lines refer to the limit $q=1$ since in this particular work we do not consider impulsive energy injection for GRBs with $q>1$. (Color online)

the same plot GRBs that correspond to the same $p$ range and astrophysical environment, to check if GRBs lie within the so-called "gray region" (Evans et al. 2009) marked in darker colors in our plots.

As seen in tables 3 and 4, the majority of GRBs do satisfy the CRs, when looking at the entire sample and when considering the lGRBs and sGRBs separately. The most fulfilled set of relations for both lGRBs and sGRBs are those of the wind SC environment for $q=0$, and $q=0.5$ for redshift (with percentages of $64.6 \%$ and $76.1 \%$, respectively), while in the case of unknown redshift for $q=0$ and $q=0.5$, again the wind slow cooling is the most populated with percentages of $89.1 \%$ and $67.2 \%$, respectively. We also note that the lowest percentages from $5.7 \%$ to $20.8 \%$ correspond to wind FC in all cases for $q=0$ and $q=0.5$ and for GRBs with unknown and known redshift. In the lower part of table 3 we consider the division into lGRBs and sGRBs for GRBs without redshift. From the percentage computed we can state that the wind slow cooling regime is the most favored $(89.1 \%$ for $q=0$ and $68.2 \%$ for $q=$ $0.5)$ for lGRBs, while the least favored is wind fast cooling (18.9\% for $q=0$ and $13.5 \%$ for $q=0.5$ ). For sGRBs, again the wind slow cooling is the most favored for $q=$ $0(87.5 \%)$, while for $q=0.5$ both ISM slow cooling and 

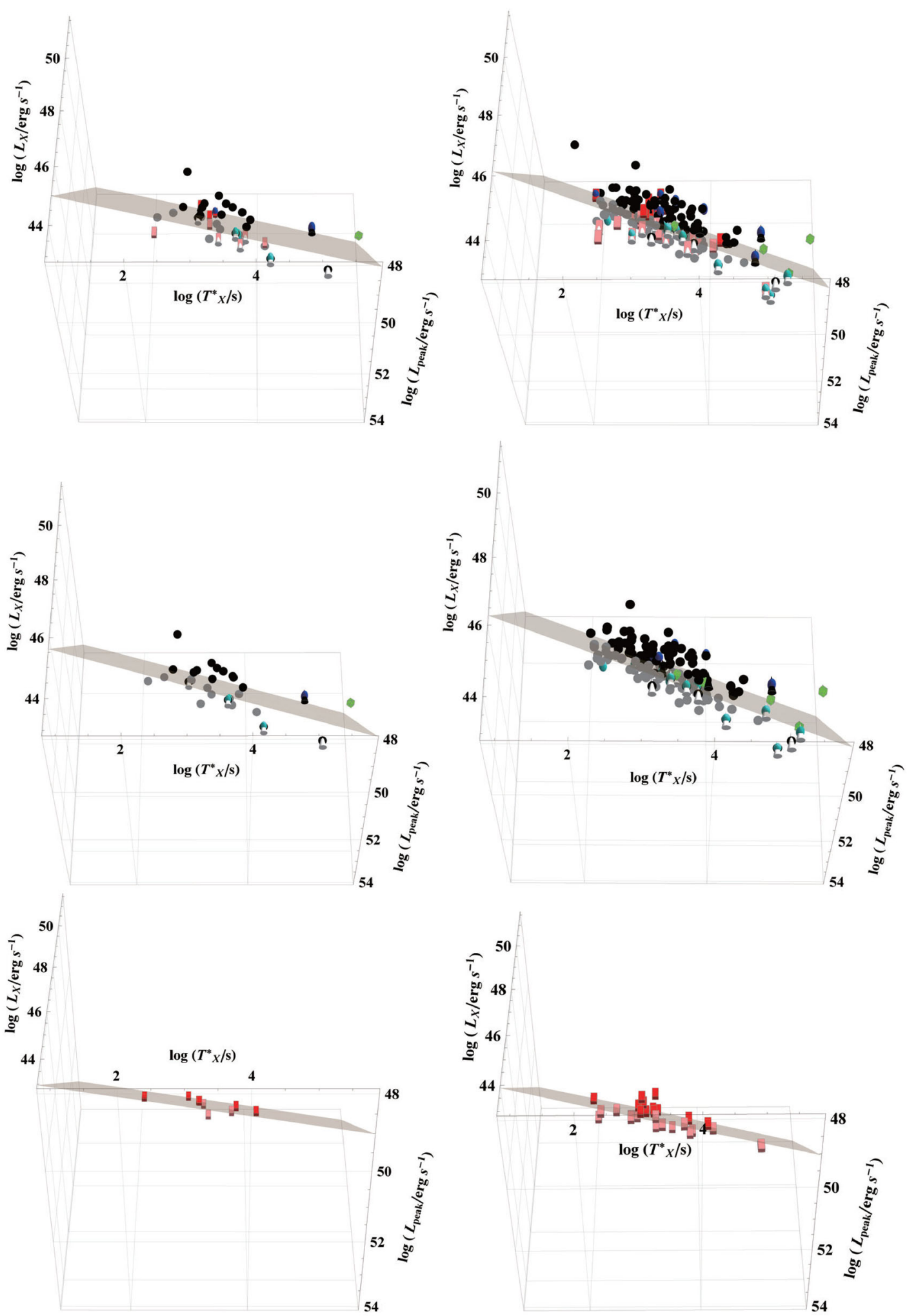

Fig. 8. GRBs are divided into all ISM for all GRBs (upper left panel), all wind for both IGRBs and sGRBs (upper right panel) groups, all ISM for IGRBs (middle left panel), all wind for IGRBs (middle right panel), all ISM for sGRBs (lower left panel), all wind for sGRBs (lower right panel) groups (see table 7), and placed on the fundamental plane defined by equation (2). The shapes and colors have been assigned as follows: GRB-SNe (black cones), XRFs (blue spheres), SEE (cuboids), IGRBs (black circles), UL GRBs (green truncated icosahedrons). The same colors, but darker, label GRBs above the plane, while lighter colors label GRBs below the plane, except for UL GRBs which are all represented by bright green truncated icosahedrons. Here, $q=0$ is considered. (Color online) 

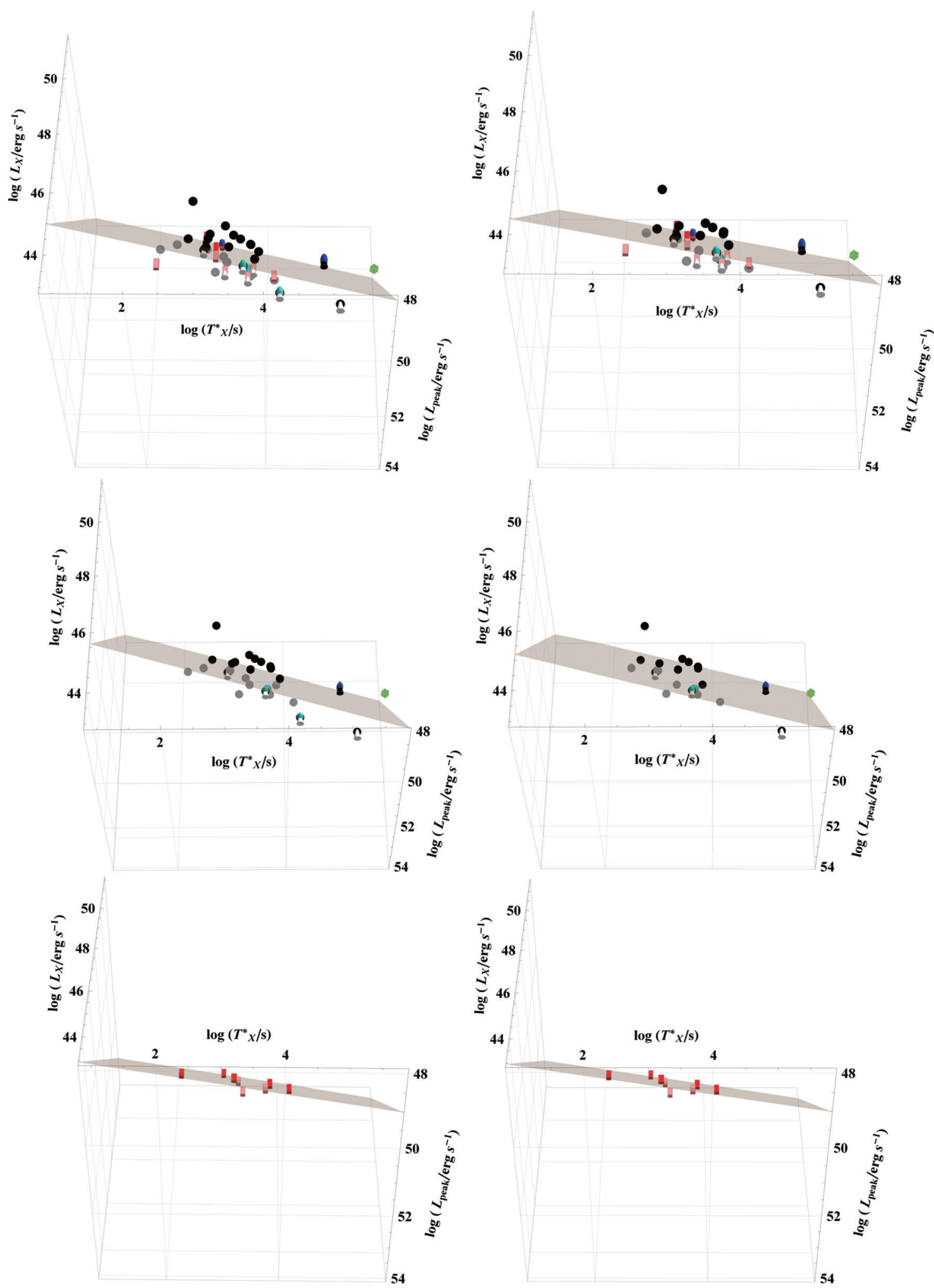

Fig. 9. As figure 8 (for $q=0$ ): GRBs are divided into ISM SC for all GRBs (upper left) and ISM FC (upper right); GRBs divided into ISM SC IGRBs (middle left) and ISM FC IGRBs (middle right); GRBs divided into ISM SC sGRBs (lower left) and ISM FC sGRBs (lower right). (Color online) 

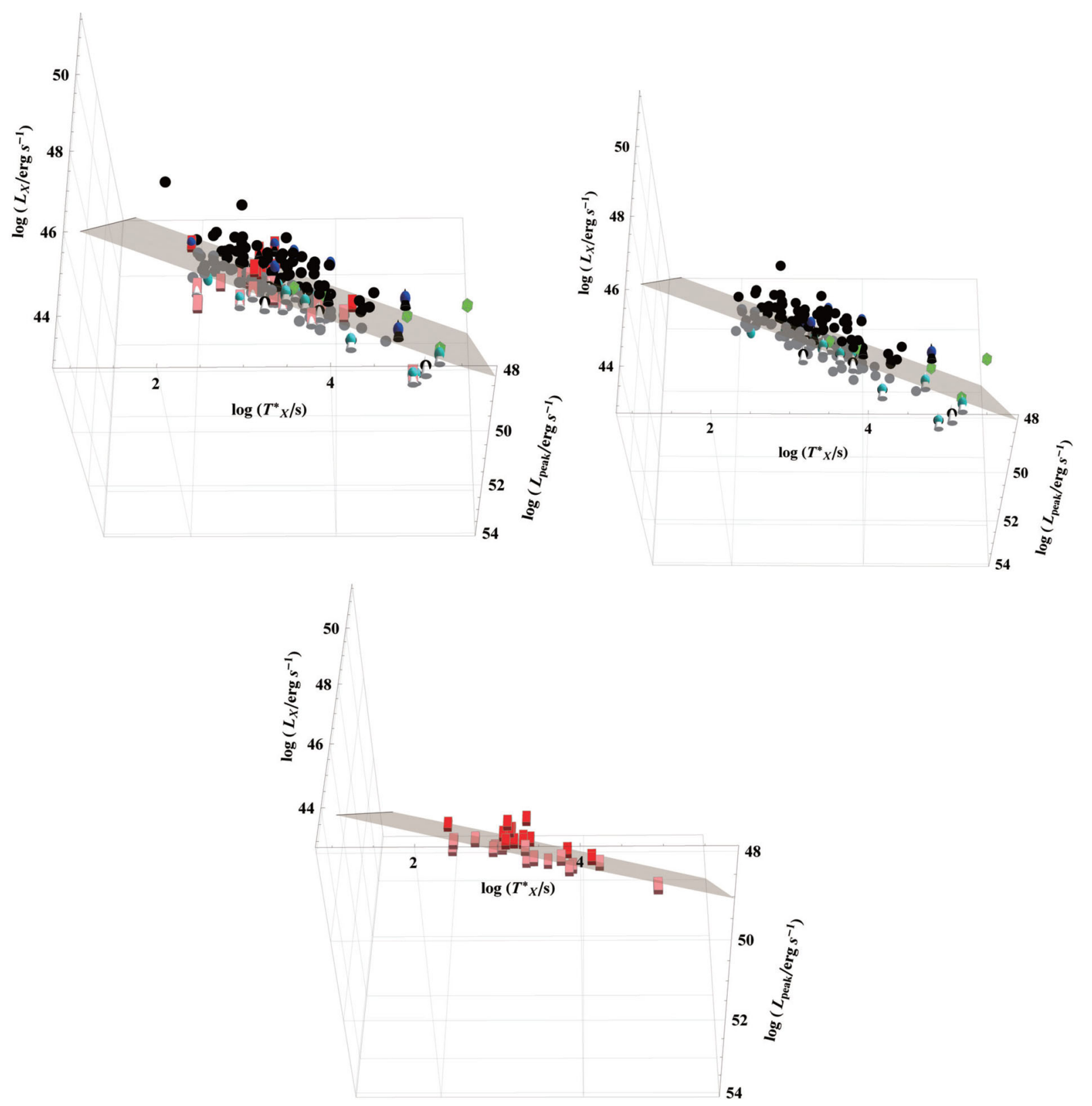

Fig. 10. As figure 8 (for $q=0$ ), considering the wind SC environment for all, IGRBs, and sGRBs. (Color online)

wind slow cooling are favored $(37.5 \%)$. Regarding the cases with redshift, we can state from looking at table 4 that both IGRBs and sGRBs favor wind slow cooling for both $q=0$ and $q=0.5$ with percentages of $64.6 \%$ and $76.1 \%$, respectively. In the lower part of the table, we investigate the cases of IGRBs, and in the bottom part of the table sGRBs. It is clear from the table that for lGRBs wind slow cooling is favored for both $q=0$ and $q=0.5$, with percentages of $89.8 \%$ and $77.4 \%$ respectively. In the case of sGRBs the most favored scenario is again wind slow cooling for both $q=0$ and $q=0.5$, with percentages of $93.5 \%$ and $67.7 \%$, respectively. As a general trend in the majority of cases, wind slow cooling is favored. We note that since some of the $p$ and $v$ ranges are repeated, the total number of GRBs is repeated for both the known redshift and unknown redshift GRBs in the following circumstances: ISM SC has the same number of GRBs as wind SC for both $q=0$ and $q=0.5$ in the sets of GRBs with known and unknown redshift. The percentages are computed under the assumption that all scenarios are equally likely to occur for each given GRB.

When grouping CRs and different GRBs, we conclude that for our sample, the ISM FC and wind FC are the least favored in the case of $p>2$ for both values of $q$ for the majority of cases. These environments are also equally disfavored in the case without redshift. 

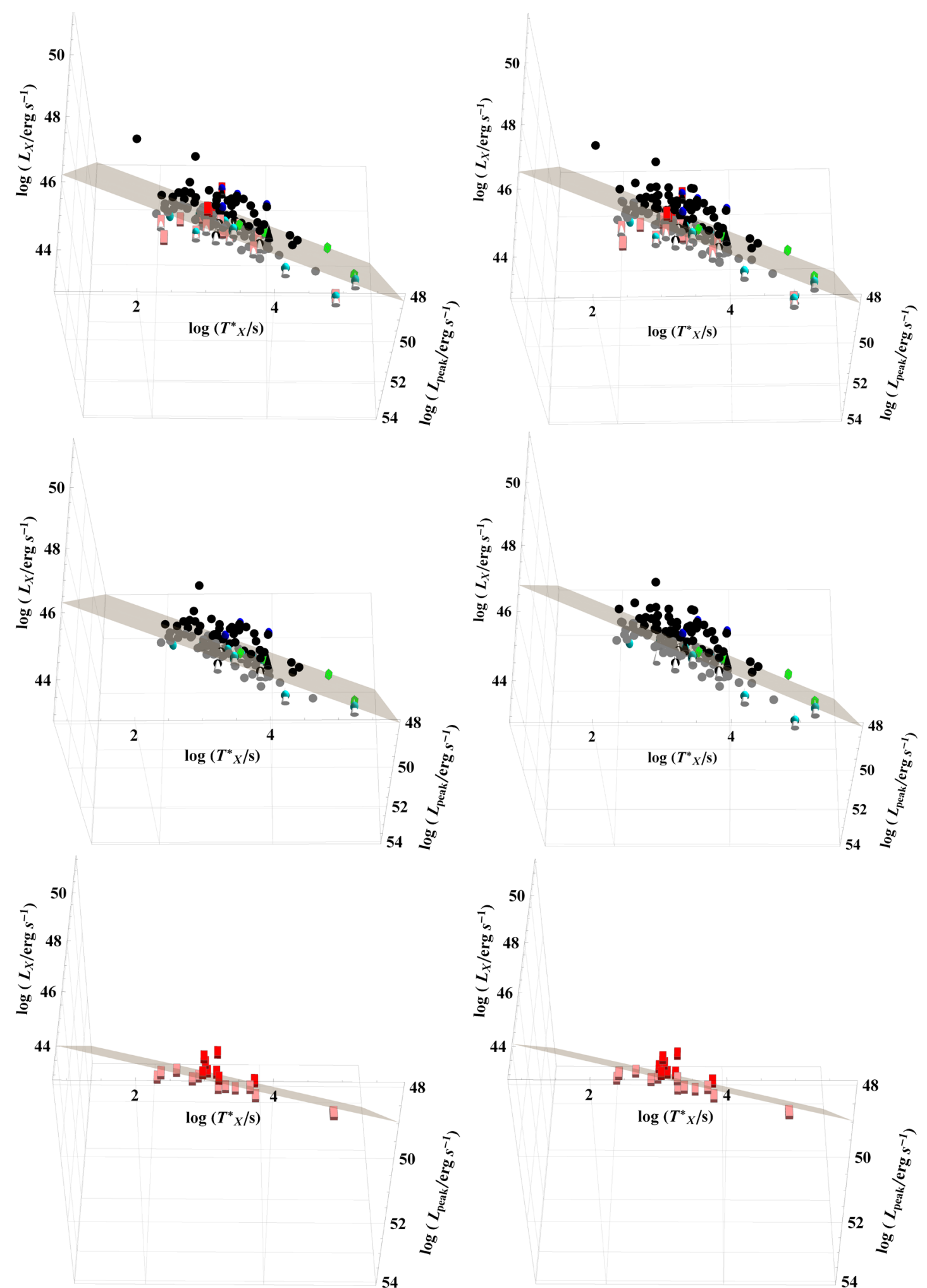

Fig. 11. GRBs divided into all ISM for all GRBs (upper left panel), all wind for all GRBs (upper right panel), all ISM for IGRBs (middle left panel), all wind for IGRBs (middle right panel), all ISM for sGRBs (lower left panel), and all wind for sGRBs (lower right panel), see table 8. These are placed on the fundamental plane defined by equation (2). The colors and symbols are the same as in figure 8. Here we consider $q=0.5$. (Color online) 

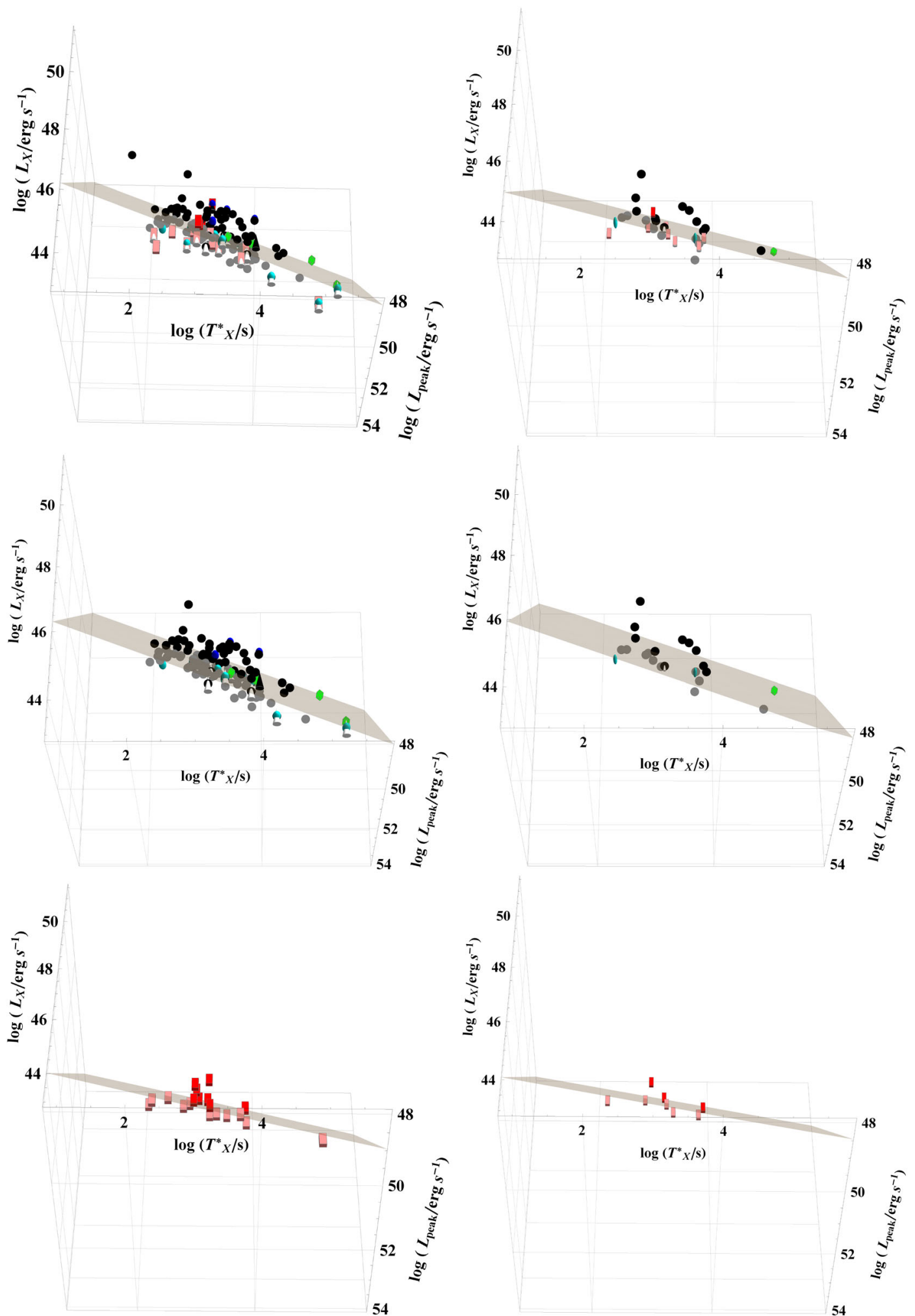

Fig. 12. As figure 11 (for $q=0.5$ ) with GRBs divided into ISM SC for all GRBs (upper left panel), ISM FC for all GRBs (lower panel), ISM SC for IGRBs (middle left panel), ISM FC for IGRBs (middle right panel), ISM SC for sGRBs (lower left panel), and ISM FC for sGRBs (lower right panel). (Color online) 


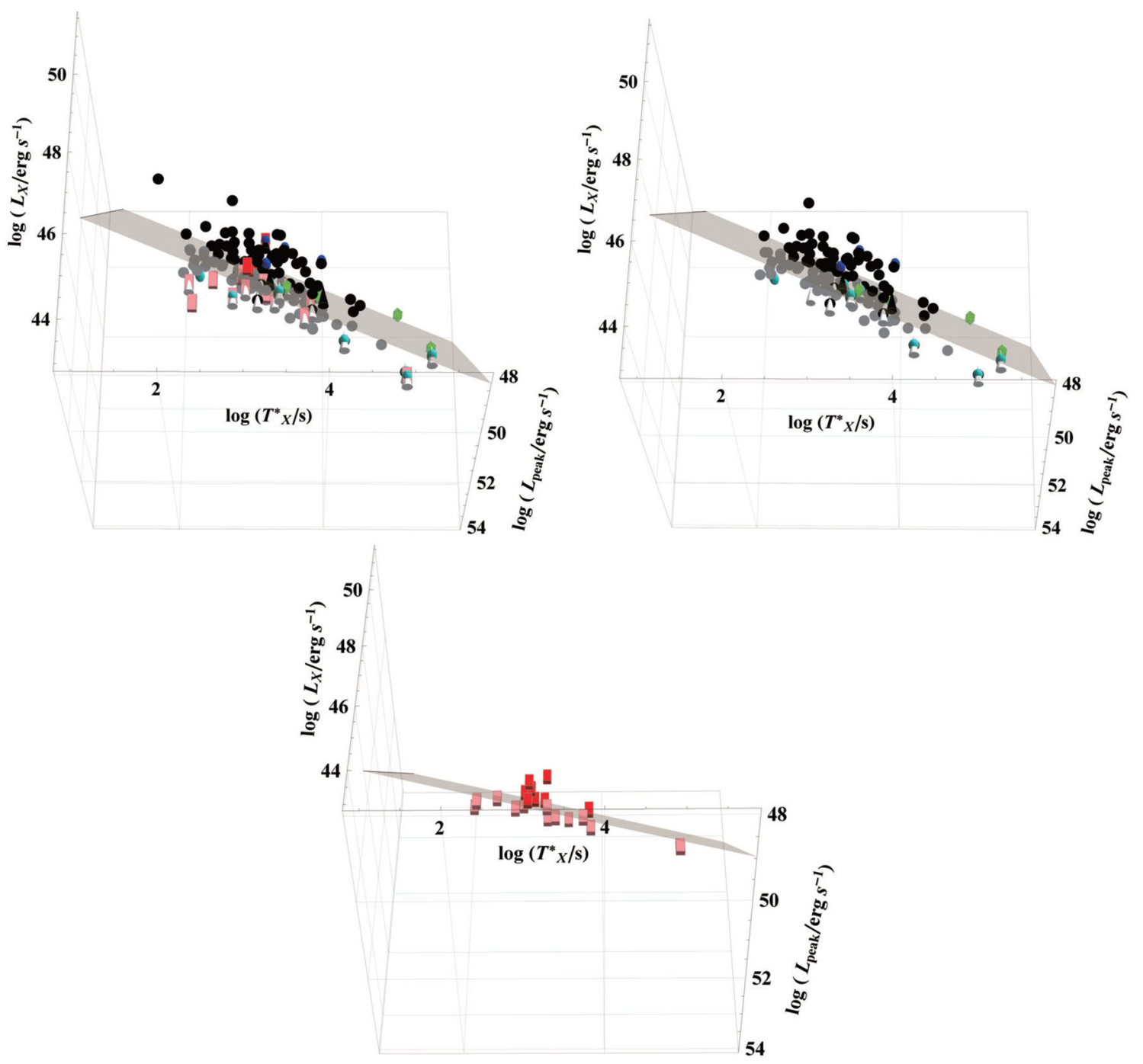

Fig. 13. As figure 11 (for $q=0.5$ ), but with GRBs belonging to wind SC environments considering the cases all, IGRBs, and sGRBs. The cases of wind FC are based on a few scattered data points, and thus their plots are omitted. (Color online)

However, the CRs should be considered a preliminary check on the robustness of the ES model without assuming any more complex physical processes. Therefore, it may still be possible that the ES model is accurate if taking into account such processes as the effects of particle acceleration in a nonlinear regime (Warren et al. 2017) or an energy injection; Zhang et al. (2006) state that the presence of phase II of the LC may be linked to three possible scenarios of continuous energy injection due to the engine at the center of GRBs. In this scenario, the fireball will take longer to decelerate due to the following:

- the long-lasting central engine expressed in equation (1);

- the different velocities of ejecta, when the slower materials accumulate and reignite the emission;

- the relevant fraction of Poynting flux of the outflow.

With regards to particle acceleration in a nonlinear regime, Warren et al. (2017) tested an evolutionary model of afterglows assuming a more complicated particle distribution than the standard power law by including a thermal population of electrons. If only a PL distribution of electrons is present, $\beta$ varies monotonically between the $v<$ $\max \left(v_{\mathrm{m}}, v_{c}\right)$ value and the $v>\max \left(v_{\mathrm{m}}, v_{\mathrm{c}}\right)$ value. The inclusion of a thermal population of electrons increases $\beta$ when the characteristic synchrotron frequency of the thermal population's exponential tail falls in a particular waveband; when the emission is once again due to the accelerated, nonthermal, population of electrons, the value of $\beta$ returns to its usual value. Such non-monotonic, soft-hard-soft behavior of $\beta$ was observed in several GRB afterglows, particularly at the beginning of the afterglow emission (Mimica et al. 2009).

However, it may also be possible that the ES model may not be the ideal scenario in some cases, and it is not external but internal shocks that produce the afterglow. Lyons et al. (2010) discussed this case as driven by a 

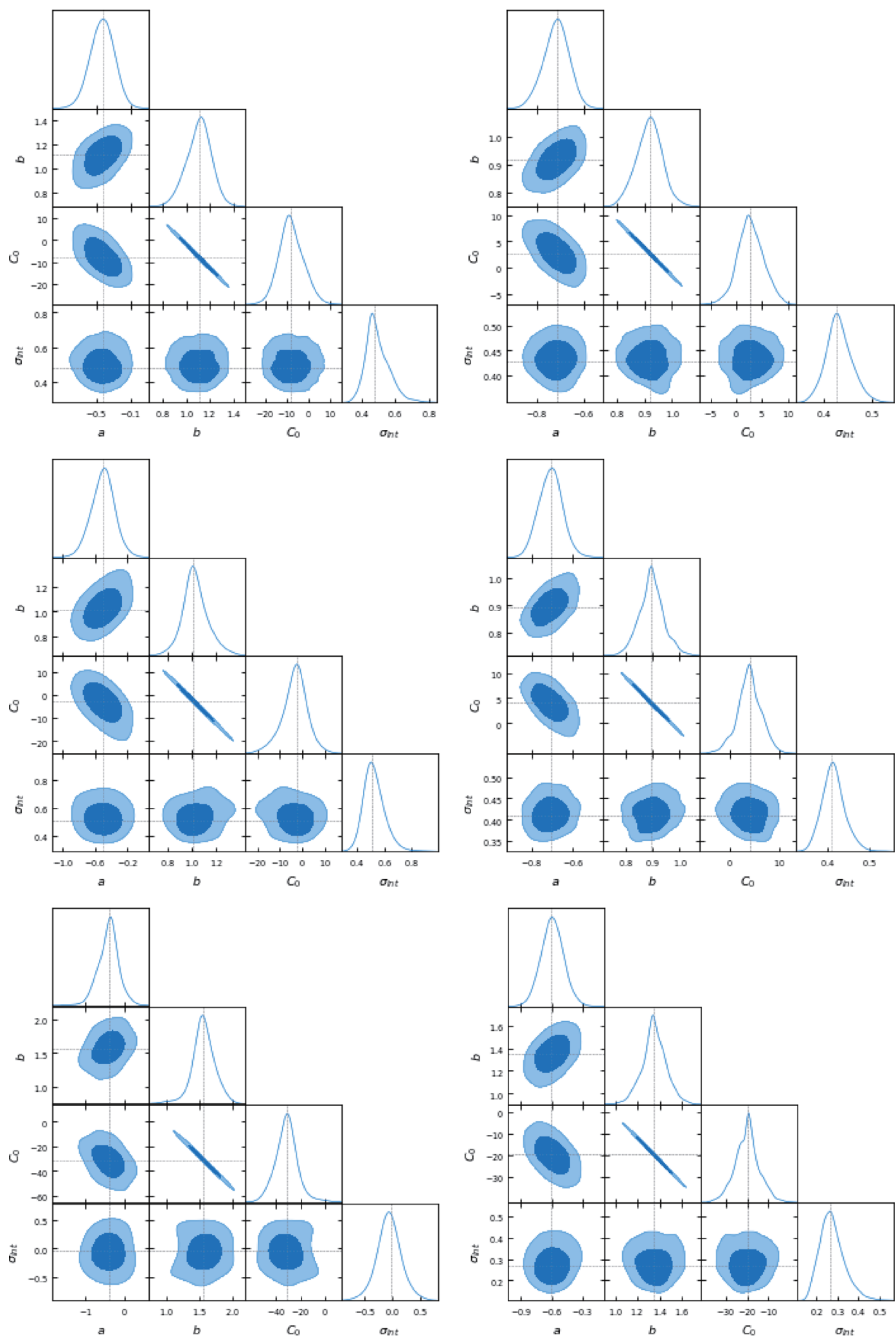

Fig. 14. Contour plots for GRB subclasses taken from table $7(q=0)$ : all ISM (IGRBs and sGRBs), all wind (IGRBs and sGRBs), all ISM (IGRBs), all wind (IGRBs), all ISM (sGRBs), and all wind (sGRBs). In these contour plots and in the following the dark blue contours indicate the $68 \%$ confidence levels, the light blue ones correspond to $95 \%$ confidence levels, and the dashed lines mark the median values of the distributions. (Color online) 

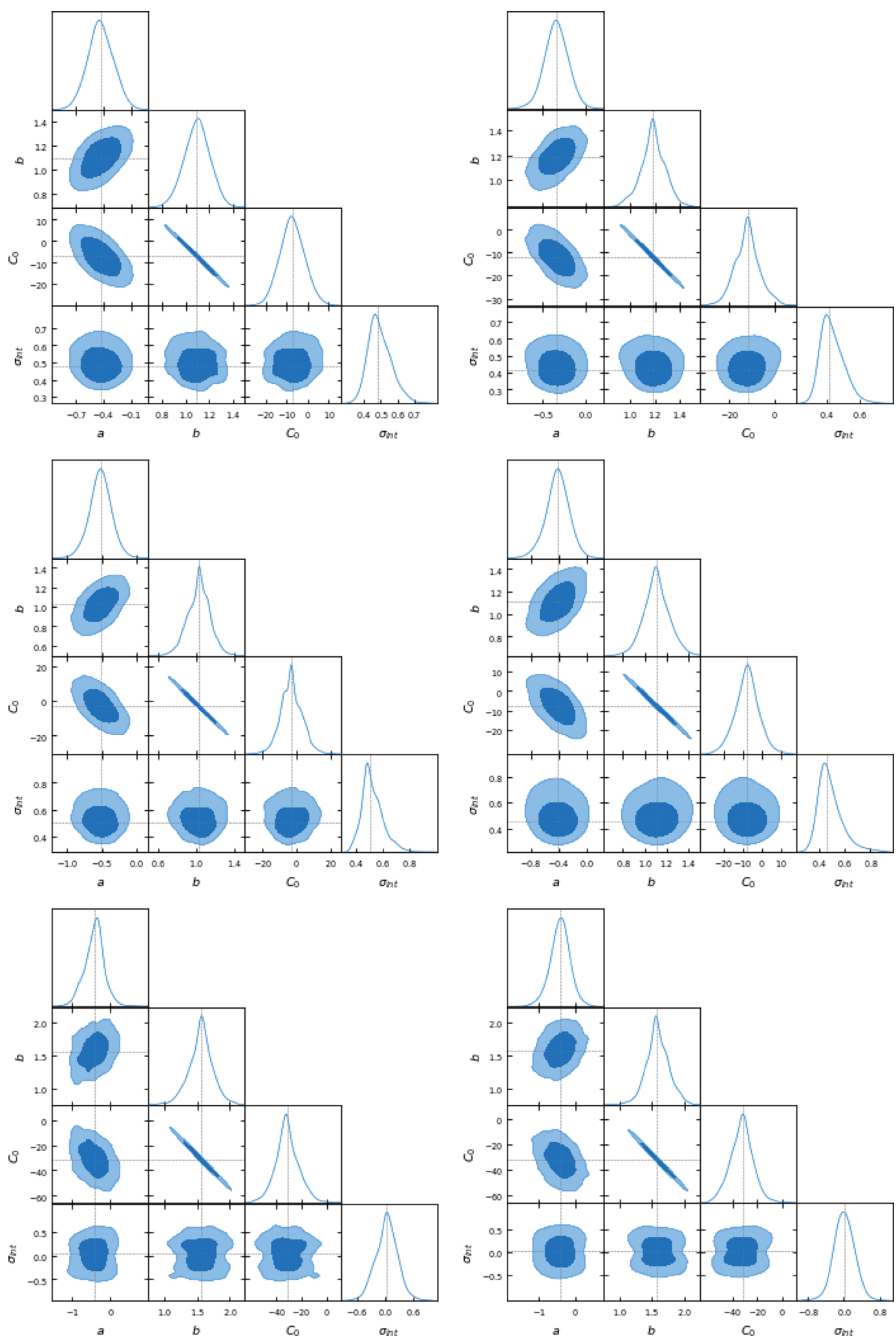

Fig. 15. Contour plots for groups taken from table $7(q=0)$ : ISM SC (all GRBs), ISM FC (all GRBs), ISM SC (IGRBs), ISM FC (IGRBs), ISM SC (sGRBs), and ISM FC (sGRBs). (Color online) 

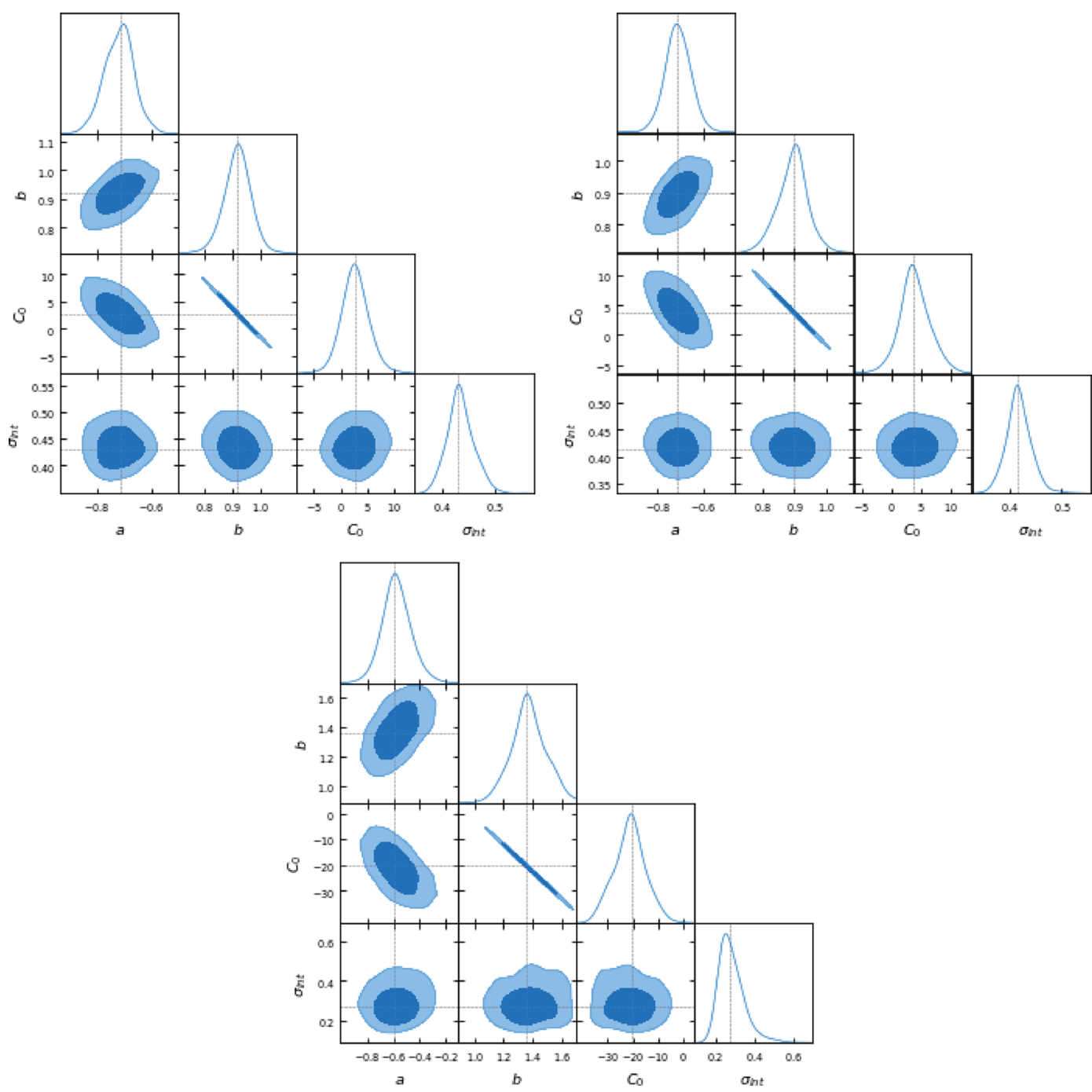

Fig. 16. Contour plots for subclasses taken from table $7(q=0)$, where wind SC is shown: all, IGRB, and sGRB cases. (Color online)

millisecond magnetar. In the ES model, the relativistic jet of the GRB is powered by a black hole formed immediately after the progenitor event. They stated that in some cases there may be a period after the progenitor event during which a millisecond magnetar forms before collapsing into a black hole. As the velocity of the magnetar diminishes over time, the engine will continually inject energy into the LC of the GRB until its collapse, which will be seen as an extremely steep decay in the LC. This feature of emission that is relatively constant followed by an abrupt decay ( $\alpha$ $\geq 4$ ) characterizes the internal PE. In our sample, we have one such GRB with $\alpha \geq 4$, GRB 061110A, and thus we have considered using the condition set by Li et al. (2018) in which they define the internal plateaus for cases with $\alpha$ $>3$. This new definition allows us to have in our sample six GRBs with internal plateaus. We now compare the results of phase II and phase III. In Srinivasaragavan et al. (2020), a similar analysis was performed on the CRs concerning phase III of the LCs, taken between times $T_{\mathrm{a}}$ and $T_{\text {end }}$. From the comparison, we see that in the current work the environments favored for $p>2$ are wind SC and ISM SC for cases $q=0$ and $q=0.5$, respectively (considering GRBs with both known and unknown redshifts). Differently from those results, in Srinivasaragavan et al. (2020) the ISM and wind FC are favored in the case of unknown redshift for $p>2$.

In the current analysis we also consider with the given values of $\alpha$ and $\beta$ the distributions of the $q$ parameters derived analytically, shown in table 6 and plotted in figure 7 . Starting from the upper left panel we show the distribution of ISM slow cooling, in the middle upper panel we present ISM with fast cooling, in the upper right panel we show wind slow cooling, and in the middle left upper panel we show the wind fast cooling for all GRBs. Then, from the middle upper central panel to the lower middle central panel we present the same environments for the lGRBs, and from 

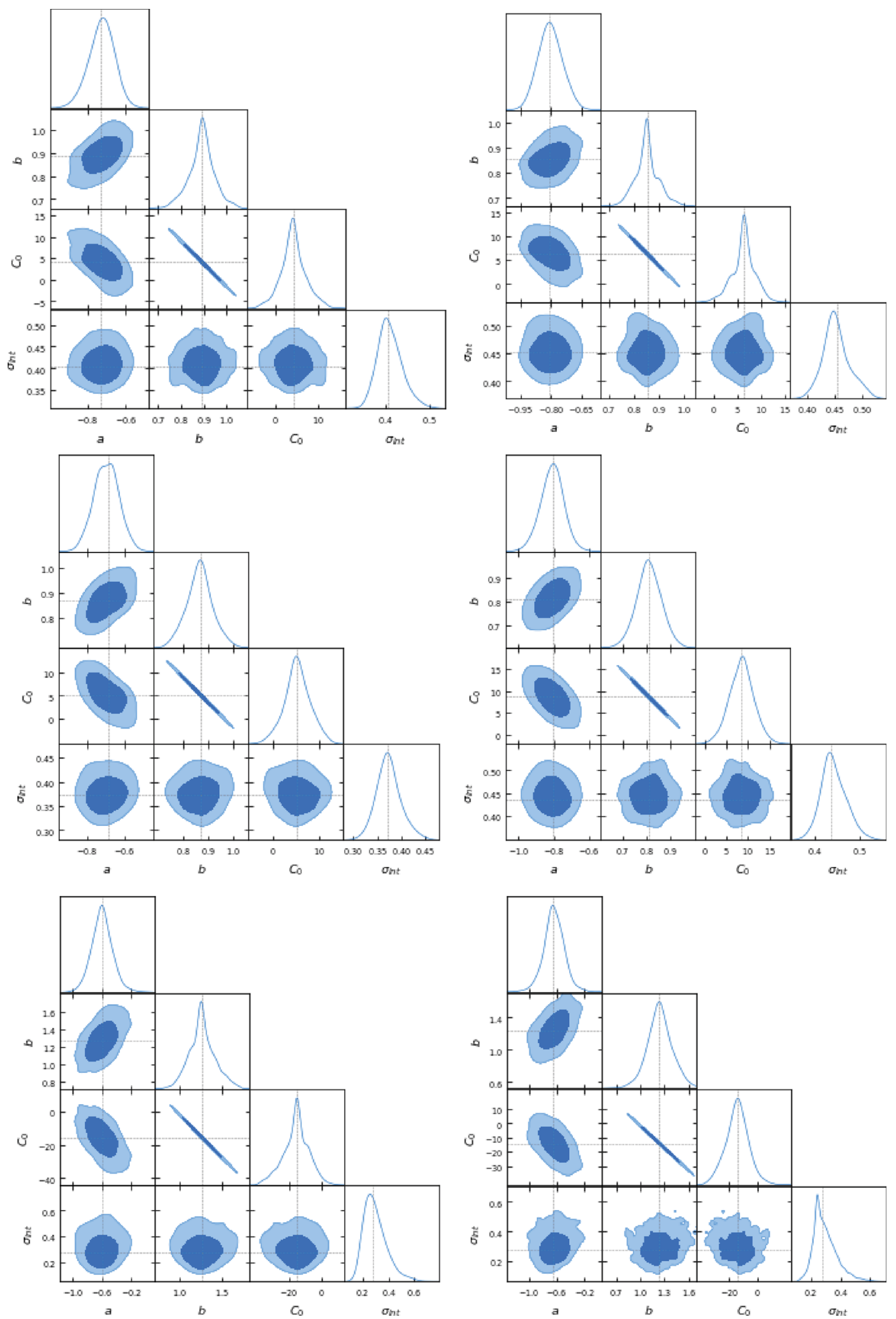

Fig. 17. Contour plots for groups taken from table $8(q=0.5)$ : all ISM (for all GRBs), all wind for all GRBs, ISM for IGRBs, wind for IGRBs, ISM for sGRBs, and wind for sGRBs. (Color online) 

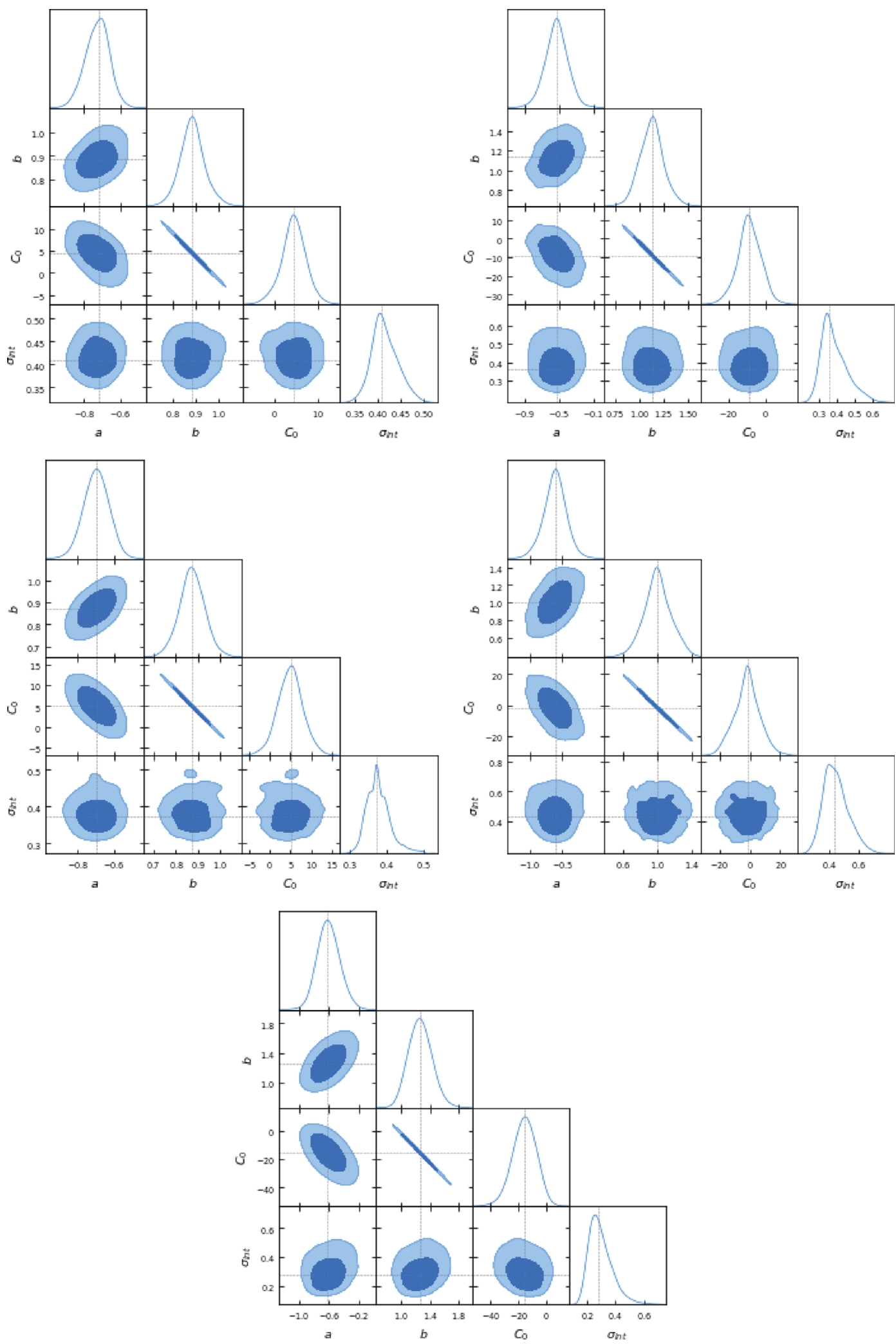

Fig. 18. Contour plots for groups taken from table $8(q=0.5)$ : ISM SC (IGRBs and sGRBs), ISM FC (IGRBs and sGRBs), ISM SC (IGRBs), ISM FC (IGRBs), and ISM SC (sGRBs). (Color online) 

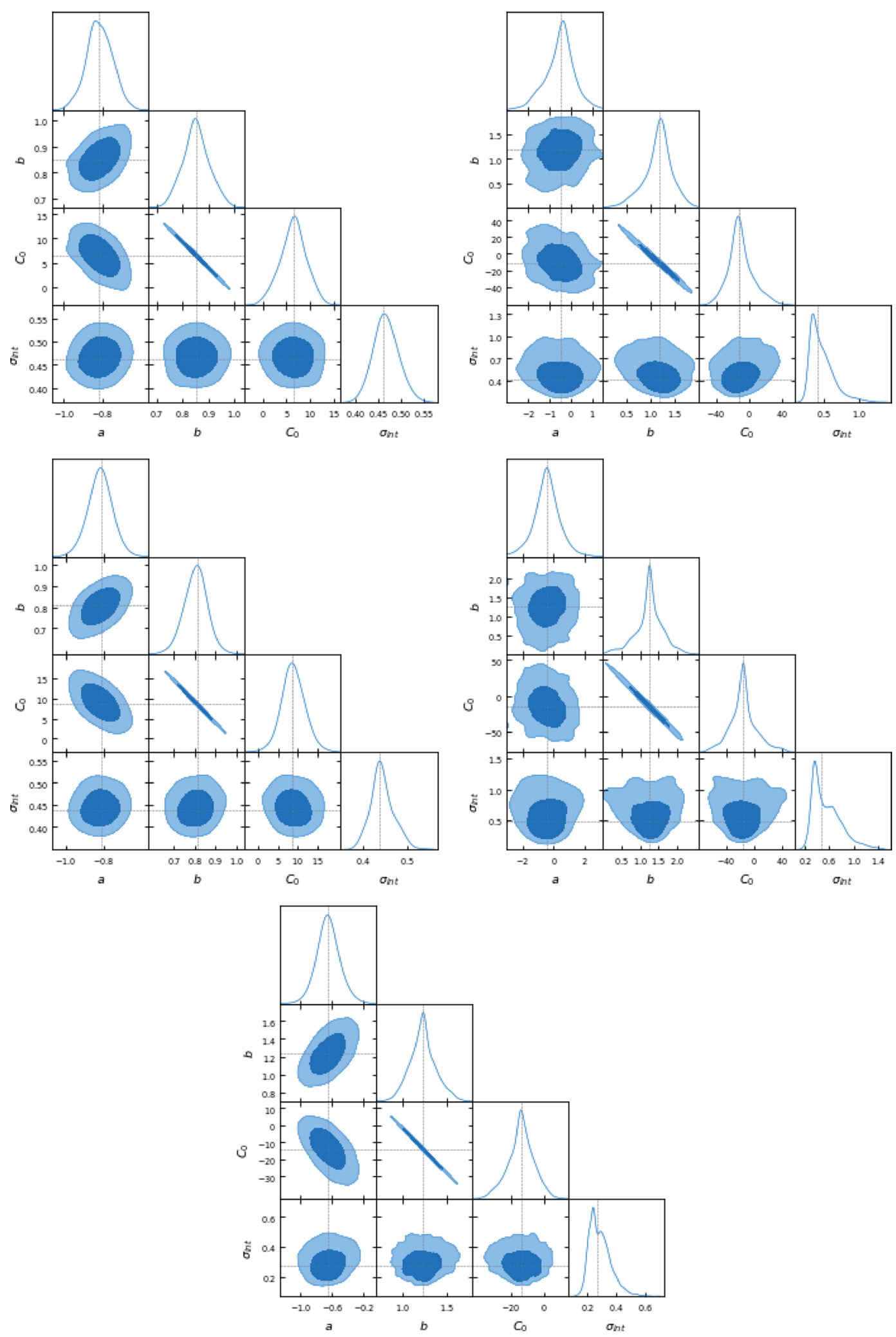

Fig. 19. Contour plots for subclasses taken from table $8(q=0.5)$ considering the wind environment: wind SC for all, wind FC for all, wind SC (for IGRBs), wind FC (for IGRBs), and wind SC (for sGRBs). (Color online) 

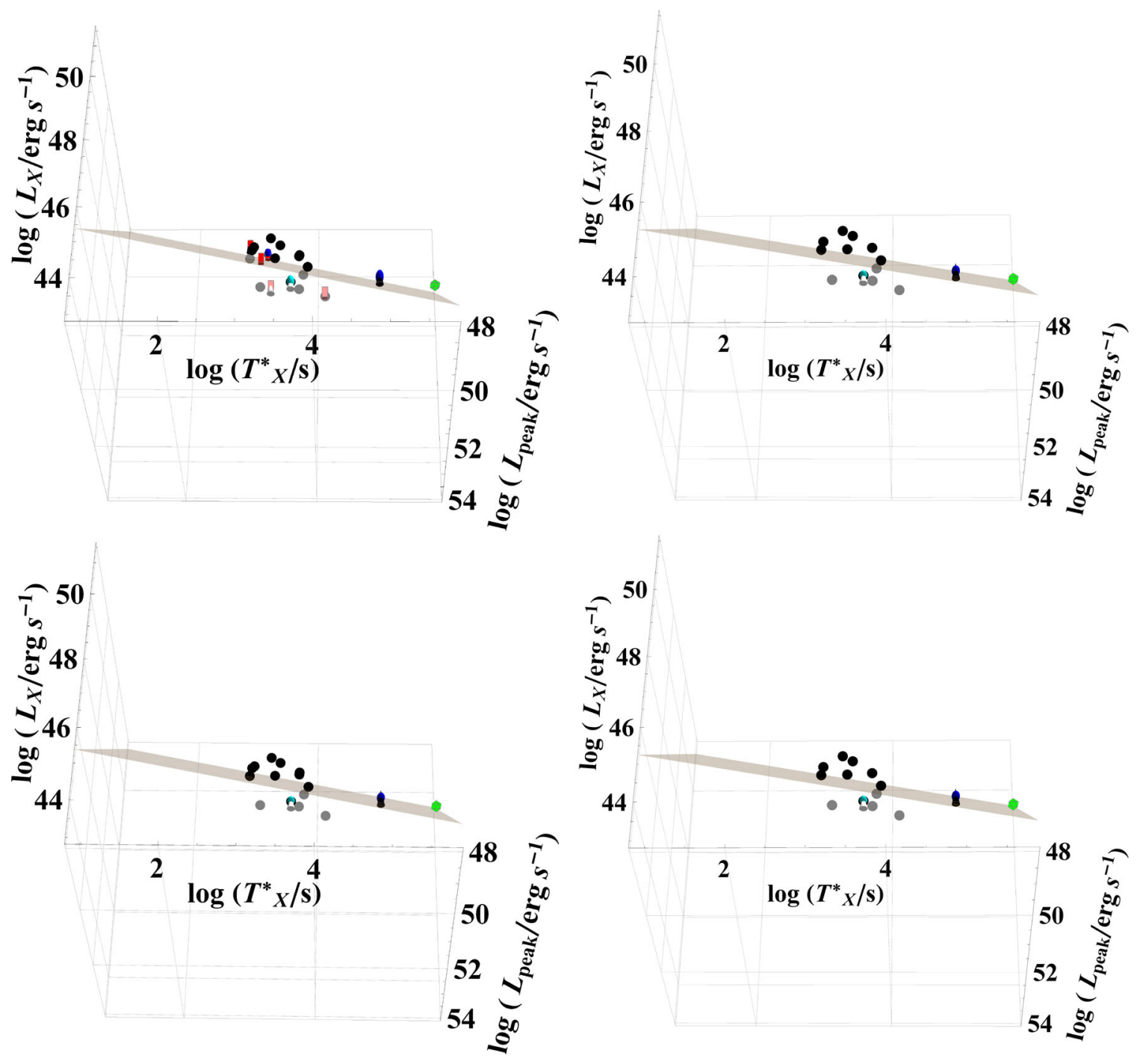

Fig. 20. Fundamental planes from equation (2) for the cases of $q$ compatible with $q=0$ reported in table 9 . The GRBs are divided into ISM SC for all GRBs (upper left panel), ISM FC for all GRBs (upper right panel), ISM SC for IGRBs (lower left panel), ISM FC for IGRBs (lower right). The colors and symbols are the same as in figure 8 . Here we consider the GRB categories for $q=0$ from table 5. (Color online)

the right lower middle panel to the lower panels we present the same configurations for the sGRBs. As we can clearly see from table 6 the error bars of the fit are sufficiently large to show that there is not a marked difference between the distributions of $q$ for sGRBs and lGRBs, since the $\mu$ values all overlap within $1 \sigma$.

\section{The 3D fundamental plane relation}

The CRs are crucial tools to pinpoint a subsample of GRBs potentially useful as standard candles. We aim to verify this property by using the 3D Dainotti relation. We fit this relation with regard to the different astrophysical groups taken from table 1, for our sample containing GRBs with known redshifts in the $\left[\log \left(T_{\mathrm{a}}\right), \log \left(L_{\text {peak }}\right), \log \left(L_{\mathrm{a}}\right)\right]$ space, applying a statistical method (D'Agostini 2005), a Bayesian approach which takes into account the error measurements in all the variables used.

A different fundamental plane is fitted for each group of GRBs. The plane equation is

$$
\log L_{a}=C_{\mathrm{o}}+a \log T_{\mathrm{a}}+b \log L_{\mathrm{peak}}+\sigma_{\mathrm{int}},
$$

where $C_{0}$ is the plane's normalization, $\sigma_{\text {int }}$ the intrinsic scatter of the sample, and $a$ and $b$ are the parameters associated with $T_{\mathrm{a}}$ and $L_{\text {peak }}$. We choose groups of GRBs that encompass: (i) all GRBs satisfying a constant-density ISM environment; (ii) all GRBs satisfying a wind environment [both points (i) and (ii) are considered regardless of the cooling regime]; (iii) an ISM environment with SC; (iv) an ISM environment with FC; (v) a wind environment with SC; 

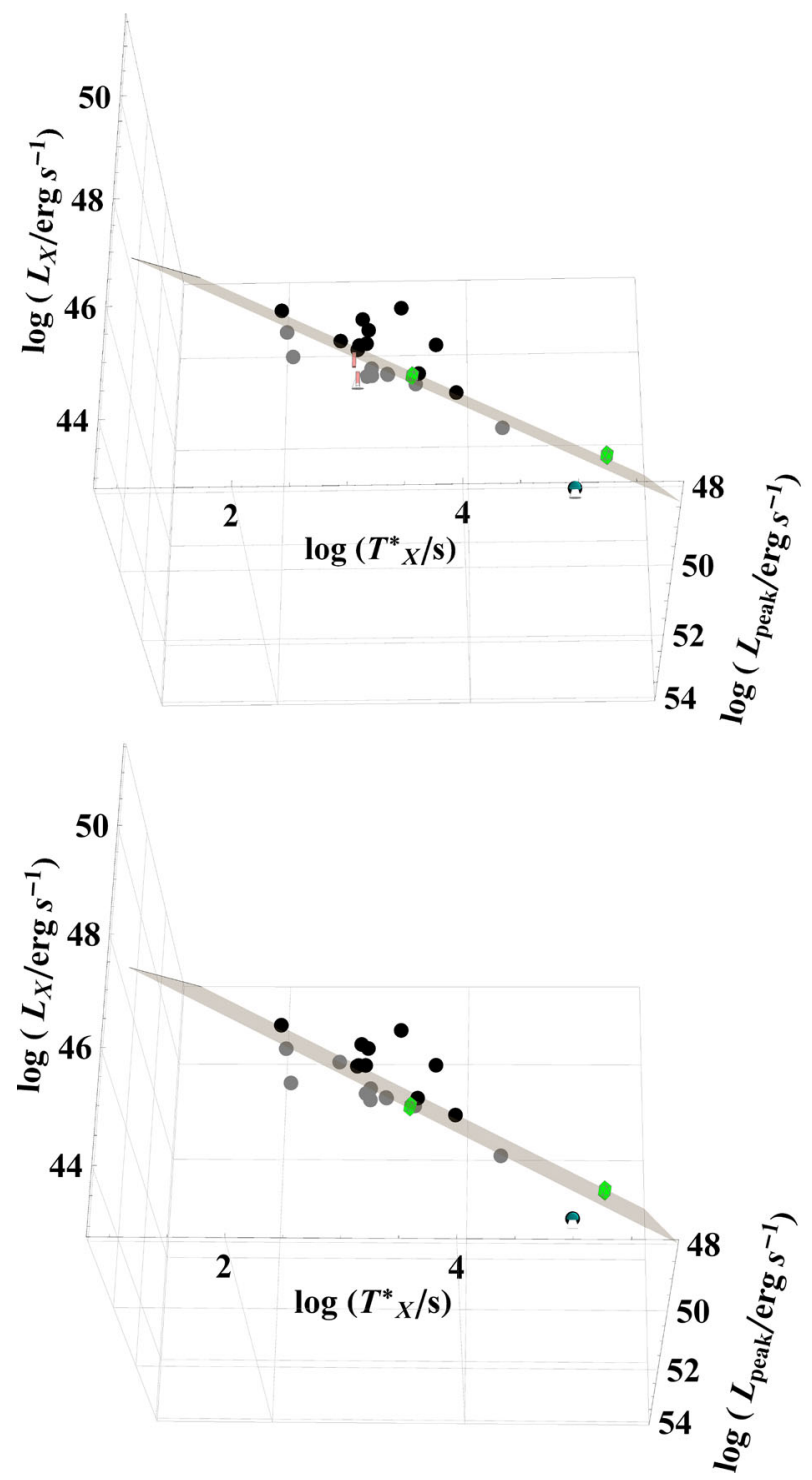

Fig. 21. GRBs from table 9, belonging to wind SC, according to the same convention as figure 20. The cases reported here are all (IGRBs and sGRBs) and IGRBs only. FC is not reported due to the lack of a significant number of points. (Color online)

(vi) a wind medium with FC. Pictures of the fundamental plane, contour plots related to their best-fitting parameters, and their tabulated values are given in figures 8-23, together with tables 7 and 8 . We investigate the parameters, in particular for the two groups used for the analysis (all ISM and all wind, and all ISM for both sGRBs and IGRBs and all wind for both sGRBs and lGRBs) and see how their best-fitting parameters compare to the rest of our sample. In the following fitting results, we will express two quantities: $R_{\text {adj }}^{2}$ and the $p$-value. The former is the square of the sample correlation coefficient between the dependent and the independent variables. The latter represents the probability that the correlation of the data is obtained by chance. For $q=0$, see table 7 for all ISM and all wind: the $a, b$, and $C_{0}$ parameters are compatible within $2 \sigma$. If we now compare ISM FC and SC we find that they are compatible within $1 \sigma$ for the $a, b$, and $C_{0}$ values. Instead, for wind SC, the $a$ parameter is compatible only within $3 \sigma$ with the ISM FC and $2 \sigma$ for ISM SC; the $b$ parameter is within $2 \sigma$ with the ISM FC and SC, while the $C_{0}$ parameter is within $2 \sigma$ for the ISM FC and for the ISM SC. As a note, the wind FC group has only three GRBs, thus preventing us from reliably fitting the plane. For $q=0$ for lGRBs, $a, b$, and $C_{0}$ are compatible within $1 \sigma$ between all ISM and all wind. For ISM FC and SC, the $a, b$, and $C_{0}$ parameters are compatible within $1 \sigma$, while the wind SC $a, b$, and $C_{0}$ are compatible within $2 \sigma$. For $q=0$ for sGRBs, the $a, b$, and $C_{0}$ parameters are compatible within $1 \sigma$ for all wind and all ISM. For ISM SC and ISM FC the $a, b$, and $C_{0}$ parameters are the same since the sGRBs that satisfy these scenarios are the same. For wind SC, ISM FC, and ISM SC all the parameters are compatible within $1 \sigma$. We now discuss the $q=0.5$ cases reported in table 8 . When we consider all GRBs, the $a, b$, and $C_{0}$ parameters are compatible within $1 \sigma$ between the all ISM and all wind categories. Considering ISM SC and ISM FC, the $a$ values are compatible within $1 \sigma$, while the $b$ and $C_{0}$ values are within $2 \sigma$. Between ISM SC and wind SC, all values are compatible within $1 \sigma$. For wind $\mathrm{SC}$ and ISM FC, the $a, b$, and $C_{0}$ values are compatible within $2 \sigma$.

Within the subclass of lGRBs, the parameter values of all ISM and all wind are compatible within $1 \sigma$ between themselves; this compatibility also holds for ISM SC and ISM FC. For wind SC and ISM SC, $a, b$, and $\mathrm{C}_{0}$ are compatible within $1 \sigma$. Considering instead wind SC and ISM FC, the same compatibility within $1 \sigma$ is observed. In the case of wind SC and wind FC, only the $a$ parameters are compatible within $1 \sigma$, while for $b$ and $C_{0}$ the compatibility is observable within $2 \sigma$. For the sGRB case with $q=0.5$, all the values of $a, b$, and $C_{0}$ are compatible within $1 \sigma$. Note that for all cases in which the fitting of the fundamental plane is not leading to reliable results due to small values of $R_{\text {adj }}^{2}$ and for a high $p$-value $>5 \%$, which indicates that the correlation induced by this environment is drawn by chance, then we discard these fittings (as indicated by dashes). Thus, we have not plotted the corresponding fundamental plane and the fitted parameters are omitted.

If we consider the analytical computation regarding the $q$ values we can refer to table 5 , in which we also present the subclasses of lGRBs and sGRBs and the corresponding environments and energy regimes. Taking the subclasses of GRBs gathered according to the results presented in table 5 and corresponding to given astrophysical environments, we have fitted the corresponding fundamental planes and derived the best-fitting parameter values presented in table 9 . 

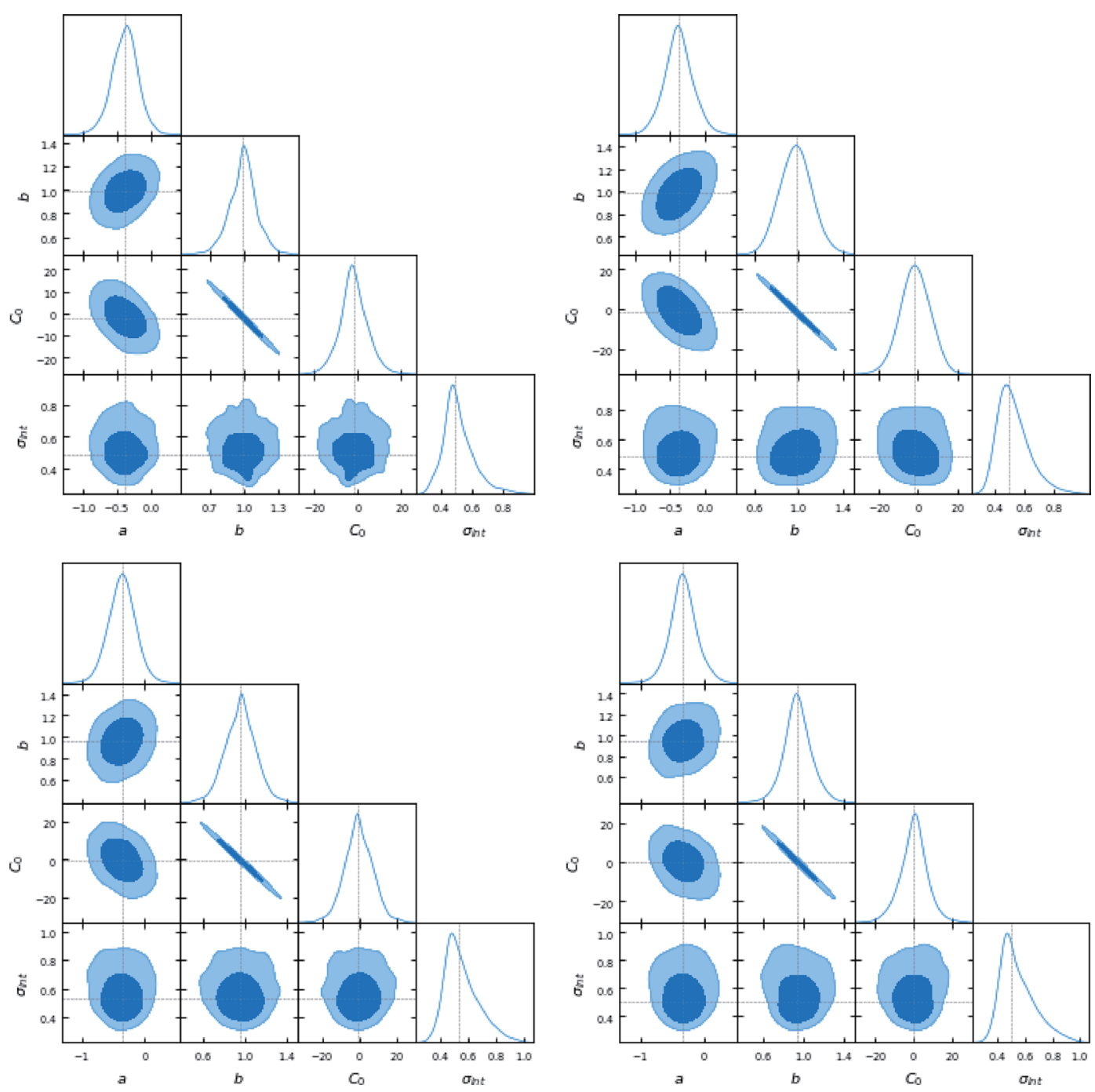

Fig. 22. Contour plots for groups taken from table $5(q=0)$. The upper left panel shows contours for the ISM SC for all GRBs, the right upper panel shows the ISM FC for all GRBs, the lower left panel shows the ISM slow cooling for IGRBs, and the lower right panel shows the ISM fast cooling for IGRBs. (Color online)

For all GRBs, the $a, b$ and $C_{0}$ parameters are compatible within $1 \sigma$ between ISM SC and ISM FC. For the wind $\mathrm{SC}$ the $a$ value is compatible with the others within $2 \sigma$, while $b$ and $C_{0}$ are compatible within $1 \sigma$. Considering the lGRB subclass only, we observe that $a, b$, and $C_{0}$ are compatible within $1 \sigma$ for ISM SC and ISM FC; wind SC, instead, presents an $a$ value compatible within $2 \sigma$ with that of ISM SC and within $3 \sigma$ with that of ISM FC, while the $b$ and $C_{0}$ values are compatible with the ISM environments within $1 \sigma$.

As an additive analysis, considering the GRBs which have a computed value of $q$ compatible with zero (see table 5), we perform the same fitting with the fundamental plane relation. The results are gathered in table 9 . In summary, for the cases of $q$ computed analytically we again find that the wind SC environment arouses interest since it is characterized by the highest number of fulfilling GRBs with a low central value of the $\sigma_{\text {int }}$ parameter.

\section{Summary and conclusions}

In summary, using GRB LCs detected by Swift (more specifically, two samples of 222 and 233 GRBs with known and unknown redshifts respectively), we consider phase II of the LCs during the time duration of the PE $\left(T_{\mathrm{t}}\right.$ to $\left.T_{\mathrm{a}}\right)$, to test both the CRs and verify the 3D Dainotti relation for our set of GRBs, to better understand the emission mechanisms of GRBs as well as their cosmological implications as potential standard candles. The majority of GRBs do satisfy the CRs as a whole, and the most fulfilled CR sets in terms of percentage are the wind SC environment $(q=0$ and $q=$ 0.5 , with and without redshift). This trend is also confirmed in the IGRB and sGRB subclasses. 

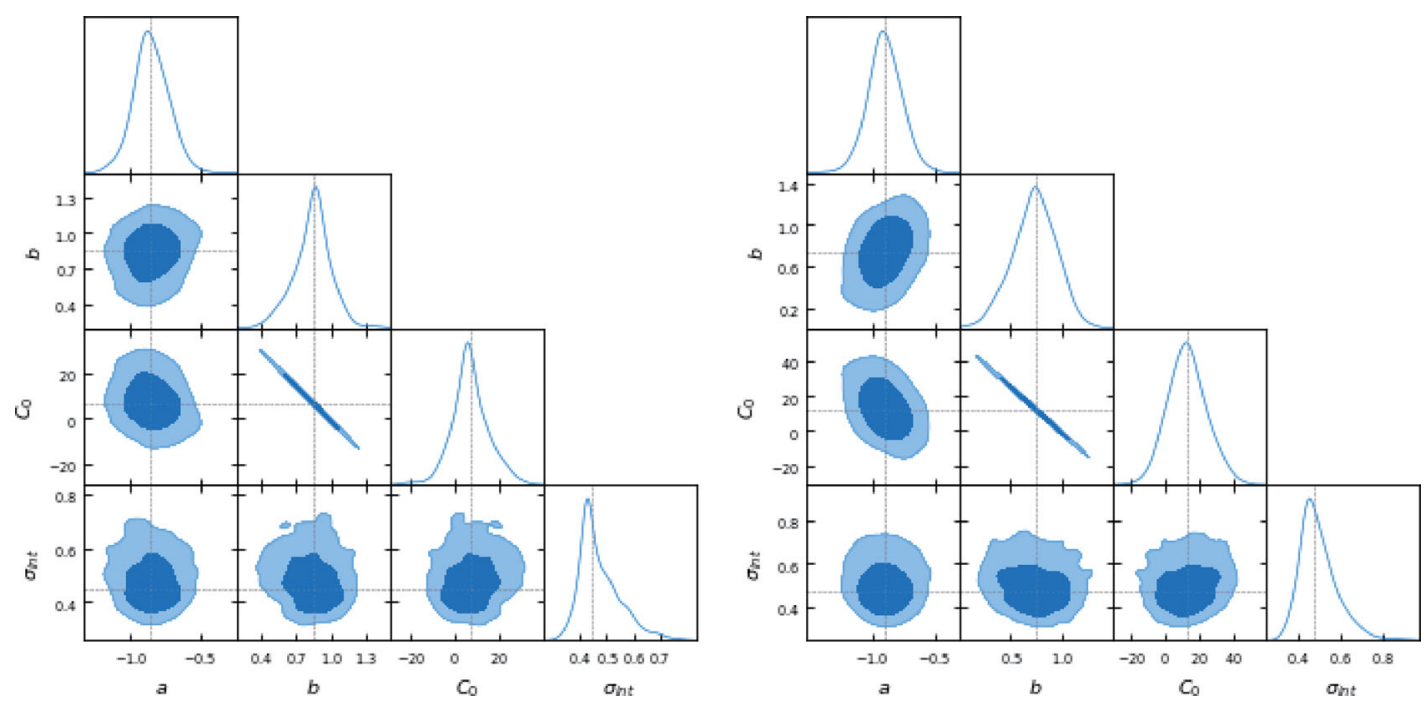

Fig. 23. Contour plots for groups taken from table $5(q=0)$. We consider wind SC for all GRBs (left panel) and wind SC for all IGRBs. (Color online)

Table 7. Best-fitting parameters for GRBs with redshift together with their number for each environment fulfilled from table 4 in the case of $q=0$.

\begin{tabular}{|c|c|c|c|c|c|c|c|}
\hline & $a$ & $b$ & $C_{0}$ & $\sigma_{\text {int }}$ & $R_{\text {adj }}^{2}$ & $p$-value & $\mathrm{N}$ \\
\hline \multicolumn{8}{|c|}{ All GRBs $(q=0)$} \\
\hline All ISM & $-0.43 \pm 0.14$ & $1.09 \pm 0.10$ & $-7.02 \pm 5.77$ & $0.46 \pm 0.07$ & 0.82 & $2 \times 10^{-10}$ & 39 \\
\hline All Wind & $-0.72 \pm 0.05$ & $0.92 \pm 0.05$ & $2.80 \pm 2.55$ & $0.44 \pm 0.02$ & 0.83 & $4 \times 10^{-60}$ & 186 \\
\hline ISM SC & $-0.43 \pm 0.14$ & $1.09 \pm 0.10$ & $-7.02 \pm 5.38$ & $0.46 \pm 0.07$ & 0.82 & $2 \times 10^{-10}$ & 39 \\
\hline ISM FC & $-0.33 \pm 0.14$ & $1.19 \pm 0.10$ & $-12.35 \pm 5.57$ & $0.39 \pm 0.08$ & 0.85 & $2 \times 10^{-9}$ & 33 \\
\hline Wind SC & $-0.72 \pm 0.05$ & $0.92 \pm 0.05$ & $2.80 \pm 2.48$ & $0.43 \pm 0.02$ & 0.83 & $4 \times 10^{-60}$ & 186 \\
\hline Wind FC & - & - & - & - & - & - & 3 \\
\hline \multicolumn{8}{|c|}{ lGRBs $(q=0)$} \\
\hline All ISM & $-0.50 \pm 0.16$ & $1.02 \pm 0.10$ & $-2.94 \pm 5.54$ & $0.48 \pm 0.08$ & 0.82 & $3 \times 10^{-9}$ & 30 \\
\hline All Wind & $-0.71 \pm 0.06$ & $0.89 \pm 0.05$ & $4.05 \pm 2.51$ & $0.41 \pm 0.02$ & 0.85 & $2 \times 10^{-55}$ & 157 \\
\hline ISM SC & $-0.50 \pm 0.16$ & $1.02 \pm 0.12$ & $-2.94 \pm 6.25$ & $0.48 \pm 0.08$ & 0.82 & $3 \times 10^{-9}$ & 30 \\
\hline ISM FC & $-0.40 \pm 0.17$ & $1.11 \pm 0.13$ & $-8.16 \pm 7.02$ & $0.42 \pm 0.09$ & 0.85 & $6 \times 10^{-8}$ & 24 \\
\hline Wind SC & $-0.71 \pm 0.06$ & $0.89 \pm 0.05$ & $4.05 \pm 2.71$ & $0.41 \pm 0.03$ & 0.85 & $2 \times 10^{-55}$ & 157 \\
\hline Wind FC & - & - & - & - & - & - & 3 \\
\hline \multicolumn{8}{|c|}{$\operatorname{sGRBs}(q=0)$} \\
\hline All ISM & $-0.41 \pm 0.26$ & $1.57 \pm 0.14$ & $-31.61 \pm 7.59$ & $0.04 \pm 0.15$ & 0.96 & 0.005 & 9 \\
\hline All Wind & $-0.60 \pm 0.10$ & $1.34 \pm 0.09$ & $-19.73 \pm 4.82$ & $0.24 \pm 0.06$ & 0.88 & $2 \times 10^{-9}$ & 28 \\
\hline ISM SC & $-0.41 \pm 0.27$ & $1.57 \pm 0.14$ & $-31.61 \pm 7.31$ & $0.04 \pm 0.16$ & 0.96 & 0.005 & 9 \\
\hline ISM FC & $-0.41 \pm 0.28$ & $1.57 \pm 0.16$ & $-31.61 \pm 8.63$ & $0.04 \pm 0.15$ & 0.96 & 0.005 & 9 \\
\hline Wind SC & $-0.60 \pm 0.11$ & $1.34 \pm 0.12$ & $-19.73 \pm 6.41$ & $0.24 \pm 0.06$ & 0.88 & $2 \times 10^{-9}$ & 28 \\
\hline Wind FC & - & - & - & - & - & - & 0 \\
\hline
\end{tabular}

Furthermore, we also test the 3D Dainotti relation for samples based on GRBs fulfilling CRs which lead to particular astrophysical environments. We find that the sGRBs for both all ISM and ISM fast cooling with $q=$ 0 have the smallest $\sigma_{\text {int }}=0.04 \pm 0.15$ in terms of the fundamental plane relation, with a probability of chance occurrence of $p=0.005$. This scatter is even smaller than the scatter found for phase III of the LCs $(\sigma=0.29 \pm$ 0.06) when we consider the same ISM FC regime, but for all GRBs (Srinivasaragavan et al. 2020). This $\sigma$ is compatible within $1 \sigma$ with the platinum sample corrected for redshift evolution and selection effects (Dainotti et al. 
Table 8. Best-fitting parameters for GRBs with redshift together with their number for each environment fulfilled from table 4 in the case of $q=0.5$.

\begin{tabular}{|c|c|c|c|c|c|c|c|}
\hline & $a$ & $b$ & $C_{0}$ & $\sigma_{\text {int }}$ & $R_{\text {adj }}^{2}$ & $p$-value & $\mathrm{N}$ \\
\hline \multicolumn{8}{|c|}{ All GRBs $(q=0.5)$} \\
\hline All ISM & $-0.73 \pm 0.06$ & $0.89 \pm 0.05$ & $4.38 \pm 2.88$ & $0.41 \pm 0.03$ & 0.81 & $4 \times 10^{-40}$ & 133 \\
\hline All Wind & $-0.85 \pm 0.07$ & $0.79 \pm 0.06$ & $9.72 \pm 3.36$ & $0.49 \pm 0.03$ & 0.77 & $7 \times 10^{-44}$ & 156 \\
\hline ISM SC & $-0.73 \pm 0.06$ & $0.89 \pm 0.05$ & $4.38 \pm 2.82$ & $0.41 \pm 0.03$ & 0.81 & $4 \times 10^{-40}$ & 133 \\
\hline ISM FC & $-0.53 \pm 0.14$ & $1.13 \pm 0.12$ & $-8.60 \pm 6.62$ & $0.36 \pm 0.08$ & 0.78 & $4 \times 10^{-7}$ & 32 \\
\hline Wind SC & $-0.81 \pm 0.06$ & $0.85 \pm 0.05$ & $6.47 \pm 2.81$ & $0.46 \pm 0.03$ & 0.80 & $2 \times 10^{-46}$ & 156 \\
\hline Wind FC & - & - & - & - & - & - & 11 \\
\hline \multicolumn{8}{|c|}{ lGRBs $(q=0.5)$} \\
\hline All ISM & $-0.70 \pm 0.07$ & $0.87 \pm 0.06$ & $5.13 \pm 2.95$ & $0.37 \pm 0.03$ & 0.84 & $8 \times 10^{-39}$ & 113 \\
\hline All Wind & $-0.81 \pm 0.07$ & $0.81 \pm 0.06$ & $8.54 \pm 3.28$ & $0.43 \pm 0.03$ & 0.82 & $1 \times 10^{-44}$ & 135 \\
\hline ISM SC & $-0.70 \pm 0.07$ & $0.87 \pm 0.06$ & $5.13 \pm 3.00$ & $0.37 \pm 0.03$ & 0.84 & $8 \times 10^{-39}$ & 113 \\
\hline ISM FC & $-0.61 \pm 0.17$ & $1.00 \pm 0.17$ & $-1.76 \pm 9.04$ & $0.38 \pm 0.09$ & 0.76 & $1 \times 10^{-6}$ & 24 \\
\hline Wind SC & $-0.81 \pm 0.07$ & $0.81 \pm 0.06$ & $8.54 \pm 3.10$ & $0.43 \pm 0.03$ & 0.82 & $1 \times 10^{-44}$ & 135 \\
\hline Wind FC & - & - & - & - & - & - & 9 \\
\hline \multicolumn{8}{|c|}{$\operatorname{sGRBs}(q=0.5)$} \\
\hline All ISM & $-0.61 \pm 0.14$ & $1.26 \pm 0.15$ & $-15.52 \pm 7.76$ & $0.23 \pm 0.08$ & 0.83 & $2 \times 10^{-5}$ & 19 \\
\hline All Wind & $-0.64 \pm 0.15$ & $1.24 \pm 0.14$ & $-14.49 \pm 7.23$ & $0.24 \pm 0.08$ & 0.82 & $8 \times 10^{-6}$ & 20 \\
\hline ISM SC & $-0.61 \pm 0.15$ & $1.26 \pm 0.16$ & $-15.52 \pm 8.25$ & $0.23 \pm 0.08$ & 0.83 & $2 \times 10^{-5}$ & 19 \\
\hline ISM FC & - & - & - & - & - & - & 8 \\
\hline Wind SC & $-0.64 \pm 0.15$ & $1.24 \pm 0.15$ & $-14.49 \pm 7.97$ & $0.24 \pm 0.08$ & 0.82 & $8 \times 10^{-6}$ & 20 \\
\hline Wind FC & - & - & - & - & - & - & 2 \\
\hline
\end{tabular}

Table 9. Best-fitting parameters for GRBs with redshift together with their number for each environment fulfilled from table 5 considering $q=0$.*

\begin{tabular}{|c|c|c|c|c|c|c|c|}
\hline & $a$ & $b$ & $C_{o}$ & $\sigma_{\text {int }}$ & $R_{\text {adj }}^{2}$ & $p$-value & $\mathrm{N}$ \\
\hline \multicolumn{8}{|c|}{ All GRBs $(q=0)$} \\
\hline ISM SC & $-0.40 \pm 0.19$ & $0.98 \pm 0.12$ & $-1.45 \pm 6.38$ & $0.45 \pm 0.10$ & 0.82 & $3 \times 10^{-5}$ & 22 \\
\hline ISM FC & $-0.37 \pm 0.20$ & $0.99 \pm 0.11$ & $-1.89 \pm 6.07$ & $0.46 \pm 0.10$ & 0.82 & $4 \times 10^{-5}$ & 20 \\
\hline Wind SC & $-0.85 \pm 0.13$ & $0.85 \pm 0.18$ & $6.87 \pm 9.52$ & $0.43 \pm 0.08$ & 0.78 & $4 \times 10^{-8}$ & 26 \\
\hline Wind FC & - & - & - & - & - & - & 0 \\
\hline \multicolumn{8}{|c|}{ lGRBs $(q=0)$} \\
\hline ISM SC & $-0.36 \pm 0.20$ & $0.96 \pm 0.13$ & $-0.28 \pm 6.81$ & $0.45 \pm 0.10$ & 0.80 & $3 \times 10^{-3}$ & 17 \\
\hline ISM FC & $-0.32 \pm 0.14$ & $0.95 \pm 0.10$ & $-0.23 \pm 5.12$ & $0.46 \pm 0.08$ & 0.80 & $4 \times 10^{-3}$ & 15 \\
\hline WIND SC & $-0.90 \pm 0.14$ & $0.72 \pm 0.20$ & $13.36 \pm 10.65$ & $0.44 \pm 0.10$ & 0.79 & $5 \times 10^{-8}$ & 24 \\
\hline WIND FC & - & - & - & - & - & - & 0 \\
\hline
\end{tabular}

*The cases of sGRBs are not reported due to the lack of a significant number of data points.

2020), where $\sigma_{\text {int }}=0.22 \pm 0.10$. Despite the estimated values of $\sigma_{\text {int }}$ for $q=0$ for the sGRBs being compatible within $1 \sigma$, it is worth noticing that the fundamental planes for sGRBs related to the all ISM environment with $q=$ 0 not only have the smallest $\sigma_{\text {int }}=0.04 \pm 0.15$ in terms of the fundamental plane relation (with a probability of occurring by chance of $p=0.005$ ), but also the smallest error bar on the same parameter. This leads to the idea that this particular environment is able to put further constraints on the determination of the intrinsic scatter of GRBs from the fundamental plane relation.

\section{Funding}

G.S. thanks the United States Department of Energy funding scheme related to the Science Undergraduate Laboratory Internship (SULI) program from which also Bowden, Wynne, and Wagner were supported. M.G.D. 
acknowledges the support from the NAOJ Division of Science and is particularly grateful to Dr. Cuellar for the support in managing the SULI students at SLAC. S.N. is partially supported by JSPS Grants-in-Aid for Scientific Research KAKENHI (A) 19H00693, Pioneering Program of RIKEN for Evolution of Matter in the Universe ( $\mathrm{r}$ EMU), and Interdisciplinary Theoretical and Mathematical Sciences Program (iTHEMS) of RIKEN. N.F. acknowledges the financial support from UNAM-DGAPA-PAPIIT through the grant IA102019.

\section{Acknowledgments}

This work used data supplied by the UK Swift Science Data Centre, University of Leicester. We thank S. Savastano and G. Sarracino for partially writing the Python codes for the D'Agostini method. We are grateful to L. Bowden, R. Wynne, R. Wagner, and Z. Nuygen for fitting some of the GRB light curves.

\section{References}

Ascenzi, S., Oganesyan, G., Salafia, O. S., Branchesi, M., Ghirlanda, G., \& Dall'Osso, S. 2020, A\&A, 641, A61

Barkov, M. V., \& Pozanenko, A. S. 2011, MNRAS, 417, 2161

Beniamini, P., Giannios, D., \& Metzger, B. D. 2017, MNRAS, 472, 3058

Beniamini, P., \& Mochkovitch, R. 2017, A\&A, 605, 1

Cannizzo, J. K., \& Gehrels, N. 2009, ApJ, 700, 1047

Cannizzo, J. K., Troja, E., \& Gehrels, N. 2011, ApJ, 734, 35

Cardone, V. F., Capozziello, S., \& Dainotti, M. G. 2009, MNRAS, 400, 775

Cardone, V. F., Dainotti, M. G., Capozziello, S., \& Willingale, R. 2010, MNRAS, 408, 1181

Chen, W., Xie, W., Lei, W.-H., Zou, Y.-C., Lü, H.-J., Liang, E.-W., Gao, H., \& Wang, D.-X. 2017, ApJ, 849, 119

D’Agostini, G. 2005, arXiv:physics/0511182

Dai, Z. G., \& Lu, T. 1998a, A\&A, 333, L87

Dai, Z. G., \& Lu, T. 1998b, Phys. Rev. Lett., 81, 4301

Dainotti, M. G., Cardone, V. F., \& Capozziello, S. 2008, MNRAS, 391, L79

Dainotti, M. G., Cardone, V. F., Piedipalumbo, E., \& Capozziello, S. 2013, MNRAS, 436, 82

Dainotti, M. G., \& Del Vecchio, R. 2017, New Astron. Rev., 77, 23

Dainotti, M. G., Del Vecchio, R., Nagataki, S., \& Capozziello, S. 2015a, ApJ, 800, 31

Dainotti, M. G., Fabrizio Cardone, V., Capozziello, S., Ostrowski, M., \& Willingale, R. 2011a, ApJ, 730, 135

Dainotti, M. G., Hernandez, X., Postnikov, S., Nagataki, S., O’Brien, P., Willingale, R., \& Striegel, S. 2017a, ApJ, 848, 88

Dainotti, M. G., Lenart, A. Ł., Sarracino, G., Nagataki, S., Capozziello, S., \& Fraija, N. 2020, ApJ, 904, 97

Dainotti, M. G., Nagataki, S., Maeda, K., Postnikov, S., \& Pian, E. 2017b, A\&A, 600, A98

Dainotti, M. G., Ostrowski, M., \& Willingale, R. 2011b, MNRAS, 418, 2202

Dainotti, M., Petrosian, V., Willingale, R., O’Brien, P., Ostrowski, M., \& Nagataki, S. 2015b, MNRAS, 451, 3898
Dainotti, M. G., Postnikov, S., Hernandez, X., \& Ostrowski, M. 2016, ApJ, 825, L20

Dainotti, M. G., Willingale, R., Capozziello, S., Fabrizio Cardone, V., \& Ostrowski, M. 2010, ApJ, 722, L215

Dall'Osso, S., Stratta, G., Guetta, D., Covino, S., De Cesare, G., \& Stella, L. 2011, A\&A, 526, A121

Del Vecchio, R., Dainotti, M. G., \& Ostrowski, M. 2016, ApJ, 828, 36

Evans, P. A., et al. 2009, MNRAS, 397, 1177

Fan, Y., \& Piran, T. 2006, MNRAS, 369, 197

Fraija, N. 2015, ApJ, 804, 105

Fraija, N., De Colle, F., Veres, P., Dichiara, S., Barniol Duran, R.,

Caligula do, E. S., Pedreira, A. C., Galvan-Gamez, A., \& Betancourt Kamenetskaia, B. 2020a, ApJ, 896, 25

Fraija, N., Laskar, T., Dichiara, S., Beniamini, P., Duran, R. B., Dainotti, M. G., \& Becerra, R. L. 2020b, ApJ, 905, 112

Fraija, N., Pedreira, A. C. C. do E. S., \& Veres, P. 2019, ApJ, 871, 200

Fraija, N., Veres, P., Beniamini, P., Galvan-Gamez, A., Metzger, B. D., Barniol Duran, R., \& Becerra, R. L. 2020c, ApJ submitted (arXiv:2003.11252)

Gehrels, N., et al. 2004, ApJ, 611, 1005

Genet, F., \& Granot, J. 2009, MNRAS, 399, 1328

Ito, H., Nagataki, S., Matsumoto, J., Lee, S.-H., Tolstov, A., Mao, J., Dainotti, M., \& Mizuta, A. 2014, ApJ, 789, 159

Izzo, L., Pisani, G. B., Muccino, M., Rueda, J. A., Wang, Y., Bianco, C. L., Penacchioni, A. V., \& Ruffini, R. 2013, in Gamma-ray Bursts: 15 Years of GRB Afterglows, ed. A. J. Castro-Tirado et al. (Les Ulis: EDP Sciences), 595

Kazanas, D., Racusin, J. L., Sultana, J., \& Mastichiadis, A. 2015, arXiv:1501.01221

Kouveliotou, C., Meegan, C. A., Fishman, G. J., Bhat, N. P., Briggs, M. S., Koshut, T. M., Paciesas, W. S., \& Pendleton, G. N. 1993, ApJ, 413, L101

Kumar, P., Narayan, R., \& Johnson, J. L. 2008, Science, 321, 376

Kumar, P., \& Panaitescu, A. 2000, ApJ, 541, L51

Kumar, P., \& Piran, T. 2000, ApJ, 532, 286

Kumar, P., \& Zhang, B. 2015, Phys. Rep., 561, 1

Levan, A. J., et al. 2007, MNRAS, 378, 1439

Levan, A. J., et al. 2014, ApJ, 781, 13

Li, L., Wu, X.-F., Lei, W.-H., Dai, Z.-G., Liang, E.-W., \& Ryde, F. 2018, ApJS, 236, 26

Liang, E., \& Zhang, B. 2006, MNRAS, 369, L37

Liang, E.-W., Zhang, B.-B., \& Zhang, B. 2007, ApJ, 670, 565

Lü, H.-J., \& Zhang, B. 2014, ApJ, 785, 74

Lü, H.-J., Zhang, B., Wei-Hua, L., Li, Y., \& Lasky, P.-D. 2015, ApJ, 805,89

Lyons, N., O’Brien, P. T., Zhang, B., Willingale, R., Troja, E., \& Starling, R. L. C. 2010, MNRAS, 402, 705

Ma, S.-B., Xie, W., Liao, B., Zhang, B.-B., Lü, H.-J., Liu, Y., \& Lei, W.-H. 2021, ApJ, 911, 97

Mazets, E. P., et al. 1981, Astrophys. Space Sci., 80, 3

Mészáros, P. 2002, ARA\&A, 40, 137

Meszaros, P., \& Rees, M. J. 1994, MNRAS, 269, L41

Meszaros, P., \& Rees, M. J. 1997, ApJ, 476, 232

Metzger, B. D., Beniamini, P., \& Giannios, D. 2018, ApJ, 857, 95

Mimica, P., Giannios, D., \& Aloy, M. A. 2009, A\&A, 494, 879

Nakar, E., Ando, S., \& Sari, R. 2009, ApJ, 703, 675 
Nakauchi, D., Kashiyama, K., Suwa, Y., \& Nakamura, T. 2013, ApJ, 778, 67

Norris, J. P., \& Bonnell, J. T. 2006, ApJ, 643, 266

Norris, J. P., Gehrels, N., \& Scargle, J. D. 2010, ApJ, 717, 411

Nousek, J. A., et al. 2006, ApJ, 642, 389

O’Brien, P. T., et al. 2006, ApJ, 647, 1213

Paczynski, B., \& Rhoads, J. E. 1993, ApJ, 418, L5

Panaitescu, A., \& Kumar, P. 2002, ApJ, 571, 779

Panaitescu, A., Kumar, P., \& Narayan, R. 2001, ApJ, 561, L171

Panaitescu, A., Mészáros, P., Burrows, D., Nousek, J., Gehrels, N., O'Brien, P., \& Willingale, R. 2006, MNRAS, 369, 2059

Panaitescu, A., Mészáros, P., \& Rees, M. J. 1998, ApJ, 503, 314

Piran, T. 2004, Rev. Mod. Phys., 76, 1143

Qin, Y., et al. 2013, ApJ, 763, 15

Racusin, J. L., et al. 2009, ApJ, 698, 43

Rea, N., Gullón, M., Pons, J. A., Perna, R., Dainotti, M. G., Miralles, J. A., \& Torres, D. F. 2015, ApJ, 813, 92

Rees, M. J., \& Mészáros, P. 1998, ApJ, 496, L1

Rhoads, J. E. 1999, ApJ, 525, 737

Rodney, S. A., et al. 2015, AJ, 150, 156

Rowlinson, A., Gompertz, B. P., Dainotti, M., O’Brien, P. T., Wijers, R. A. M. J., \& van der Horst, A. J. 2014, MNRAS, 443, 1779

Rowlinson, A., O’Brien, P. T., Metzger, B. D., Tanvir, N. R., \& Levan, A. J. 2013, MNRAS, 430, 1061

Sakamoto, T., et al. 2007, ApJ, 669, 1115

Sari, R., \& Mészáros, P. 2000, ApJ, 535, L33

Sari, R., \& Piran, T. 1995, ApJ, 455, L143

Sari, R., \& Piran, T. 1999a, ApJ, 517, L109

Sari, R., \& Piran, T. 1999b, A\&AS, 138, 537

Sari, R., Piran, T., \& Narayan, R. 1998, ApJ, 497, L17

Soderberg, A. M., et al. 2006, Nature, 442, 1014
Srinivasaragavan, G. P., Dainotti, M. G., Fraija, N., Hernandez, X., Nagataki, S., Lenart, A., Bowden, L., \& Wagner, R. 2020, ApJ, 903, 18

Stratta, G., Dainotti, M., Dall'Osso, S., Hernandez, X., \& De Cesare, G. 2018, ApJ, 859, 155

Stratta, G., et al. 2013, ApJ, 779, 66

Tang, C.-H., Huang, Y.-F., Geng, J.-J., \& Zhang, Z.-B. 2019, ApJS, 245,1

Toma, K., Ioka, K., Sakamoto, T., \& Nakamura, T. 2007, ApJ, 659, 1420

Troja, E., et al. 2007, ApJ, 665, 599

Uhm, Z. L., \& Zhang, B. 2014, ApJ, 780, 82

Wang, X. G., et al. 2015, ApJS, 219, 9

Warren, D. C., Beauchemin, C. A. A., Barkov, M. V., \& Nagataki, S. 2021, ApJ, 906, 33

Warren, D. C., Ellison, D. C., Barkov, M. V., \& Nagataki, S. 2017, ApJ, 835, 248

Willingale, R., et al. 2007, ApJ, 662, 1093

Willingale, R., Genet, F., Granot, J., \& O’Brien, P. T. 2010, MNRAS, 403, 1296

Zhang, B., et al. 2007, ApJ, 655, 989

Zhang, B., Fan, Y. Z., Dyks, J., Kobayashi, S., Mészáros, P., Burrows, D. N., Nousek, J. A., \& Gehrels, N. 2006, ApJ, 642, 354

Zhang, B., \& Mészáros, P. 2001, ApJ, 552, L35

Zhang, B.-B., et al. 2011, ApJ, 730, 141

Zhang, B.-B., Zhang, B., Murase, K., Connaughton, V., \& Briggs, M. S. 2014, ApJ, 787, 66

Zhao, L., Liu, L., Gao, H., Lan, L., Lei, W., \& Xie, W. 2020, ApJ, 896,42

Zhao, L., Zhang, B., Gao, H., Lan, L., Lü, H., \& Zhang, B. 2019, ApJ, 883, 97 